\title{
THE COMPLEX LORENZ EQUATIONS
}

\author{
A. C. FOWLER \\ Department of Mathematics, Massachasetts Institute of Technology, Cambridge. Mass. 02139. USA
}

\section{J. D. GIBBON}

Depariment of Mathematics. Imperial College of Science \& Technology. London SW7 2BZ, GB

and

\section{J. MCGUINNESS}

Department of Mathematical Physics, University College Dublin. Belfield, Dublin 4, Ropublic of Ireland

Received II March 1981

We tave under:aken a study of the complex Lorenz equations

$$
\begin{gathered}
\dot{x}=-v x+a y . \\
\dot{y}=(r-z) x-a y, \\
z=-b z+\frac{b}{b}\left(x^{*} y+x y^{*}\right)
\end{gathered}
$$

where $x$ and $y$ are complex and $z$ is real. The complex parameters $r$ and $a$ are defined by $r=r_{1}+i r_{2}: a=1-i e$ and $\sigma$ and $b$ are real. Behaviour remarkably different from the real Lor nz model occurs. Only the origin is a fixed point except for the special case $e+r:=0$. W'e have been able to determine analytically two critical values of $r_{1}$, namely $r_{10}$ and $r$ íc The origin is a stable fixed point for $0<r_{1}<r_{1}$, but for $r_{1}>r_{1}$ a Hopf bifurcation to a linit cycle occurs. We have an exact analytic solution for this limit cycle which is always stable if $\sigma<b+1$. If $a>b+1$ then this limit is only stable in the region $r_{i c}<r_{1}<r_{i}$. When $r_{1}>r l_{e}$, a transition to a finite amplitude oscillation about the limit cycle occurs. The naturs of this bifureation is studied in detail by using a multiple time scale analysis to derive the Stuart-Landau amplitude equation from the original equations in a frame rotating with the limit cycle frequency. This latter bifurcation is either a sub- or super-critical Hopf-like bifurcation to a doubly periodic motion, the direction of bifurcation depending on the parameter values. The nature of the bifureation is complicated by the existence of a zero eigenvalue.

\section{Introduction}

In a previous paper [1], the so-called "complex" Lorenz equations were derived as a generalization of the original equations first derivec by Lorenz [2]. The complex equations are written in the form

$$
\begin{aligned}
& \dot{x}=-o x+\sigma y, \\
& \dot{y}=-x z+r x-a y, \\
& \dot{z}=-b z+\frac{t}{2}\left(x^{*} y+x y^{*}\right) .
\end{aligned}
$$

The Rayleigh number $r$ and the parameter $a$ are complex numbers defined by

$$
\begin{aligned}
& r=r_{1}+\mathrm{i} r_{2}, \\
& a=1-\mathrm{i} e .
\end{aligned}
$$

and $\sigma_{1} b, r_{1}, r_{2}$ and $e$ are real and positive. The form of (1.1) shows that $x$ and $y$ are complex but $z$ is real.

The ntention of this paper is to make a mathematical study of eqs. (1.1) in order to show that significantly difierent behaviour occurs in the bifurcation sequence than in the 
real Lorenz equations. The real Lorenz model is embedided in (1.1) and can be rucovered when $r_{2}=e=0$ and $x$ and $y$ are real. The physical motivation for studying the complex Lorent model comes from work by two of the three prese- iuthors who derived a set of amplitude equations near criticality for a class of dis. persively unstable, weakly nonlinexr, weakly damped physical systems $[1,4]$. This was sased on work which :ons dered the und inped systems first [3] anc then seak dampin was added afterwards.

When only temporal variation was included. the relevant set of amplitude equations turned out to be of the form

$\frac{\mathrm{d}^{2} A}{\mathrm{dT}^{2}}+\Delta_{1} \frac{\mathrm{d} A}{\mathrm{~d} T}=\alpha A-\beta A B$.

$\frac{\mathrm{d} B}{\mathrm{~d} T}+\Delta_{2} B=\frac{\mathrm{d}}{\mathrm{d} T}|A|^{2}+\Delta_{3}|A|^{2}$.

In the circumstarces discussed in [1]. the parameters $\beta, i_{2}$ and $\Delta_{3}$ were always real and positive. If only weak damping is added to the original system then $\alpha$ and $\Delta_{1}$ are also real and positive. This is the case for the laser equations $[1,4]$. If cxtra weak dispersive effects are also added then it turns out that $\alpha$ and $\Delta_{1}$ become complex. This circumstance occurs in the 2layer and Eady models of baroclinic instability $[1,5$, when a "weak" beta-effect is included. Eqs. (1.3) can be transformed into eqs. (1.1) by the following transformations:

$t=\Omega T$.

$\Omega=\operatorname{Re}\left(\Delta_{1}\right)-\Delta_{1} / 2$

$x=(2 \beta)^{1 / 2} \Omega^{-1} A$.

$z=2 \beta \Omega^{-1} \mathrm{~A}^{-1} \mathrm{~B}$.

where the variables $r$, or $a$ and $b$ in (1.1) are given by

$c r=J_{3} / 2 \Omega$.

$s=j, \Omega$.

$$
\begin{aligned}
& r_{1}=1+2 \operatorname{Re}(\alpha) /\left(\Delta_{1} \Omega\right) . \\
& r_{:}=\left[2 \operatorname{Im}(\alpha)+\Delta_{1} \operatorname{Im}\left(\Delta_{1}\right)\right] /\left(\Omega \Delta_{3}\right), \\
& r=-\ln \left(\Delta_{1}\right) \Omega .
\end{aligned}
$$

The complex Lorenz lations also form the basic model for bistable optical systems of two level atoms. Hassan. Drumriond and Walis [19] and Ikeda [20] have shown that in a single mode high-Q ring cavity. the semi-classical equations of motion are a set of damped Maxwell-Bloch type equations. By coservation we can show that these can be transformed into eqs. (1.1), but with an external driving field. In this case, $r_{z}=0$. but $e \neq 0$. Refs. 19 and 20 extend earlier results by Bonifacio and Lugiato [21]. As a physical problem, the driving field is necessary ar obviously the inclusion of this adds an extra degree of cumplication to the problem. A study of the system (1.1) on its own is therefore important as a first step towards understanding the more general problem, in which a forcing term is present.

However, the essence of this paper is not a discussion of the physical derivation of (1.1) since this was performed in [1] but rather we seek to undertake a mathematical and simple numerical analysis of these equations in their own right. A consideration of this more general system may also cast more light on the real Lorenz model.

Our approach is analytical, but we should point cut that the analytical results obtained here were strongly motivated by the results of numerical computations. An example of this is the exact periodic solution displayed in section 2. the form of which was suggested by numerical compurations which gave a perfect ellipse in the $\operatorname{Re}(x)-\operatorname{Re}(y)$ phase plane with $z$ quickly reaching a constant equilibrium value. The analysis of the bifurcation of the limit cycle to doubly periodic solutions given in section 3 was also suggested by numerical results which showed fast and slow oscillations, thereby indicating that a multiple time scale calculation 
was in ordet. It is interesting, in our view, that so much info: mation can be derived by analytical means for what is essentially a fifth order problem. A qualitative difference between the first two bifurcations is that while the first is a supercritical Hopf bifurcation of the origin into a limit cycle, the nature of the second is blurred by the existence of a $\lambda=0$ eigenvalue which occurs because of rotational symmetry. For this reason, the atims of sections 2 and 3 are different. In section 2, jollowing Loren: in which we use $r_{1}$ as the inair bifurcation parameter, we calculate the two critical values of $r_{1}$ at which firstly, the origin bifurcates to the limit cycle and secondly, at which this limit cycle becomes unstable. Section 3 is devoted to understanding the nature of this bifurcation using multiple scales to obtain the Stuart-Landau equation in the rotating frame,

$\frac{d A}{d t}=k_{1} A+k_{2} A|A|^{2}$

Following McLaughlin and Martin [6], the criterion for determining whether the bifurcation is super- or sub-critical is determined by whether $\operatorname{Re}\left(k_{2}\right)$, is negative or positive respecively and consequently whether the limit cycle undergoes "soft' bifurcation to a doubly periodic solution. or 'hard" bifurcation to some other type of motion.

\section{An exact periodlc solution}

In studying (1.1) we shall follow Lorenz' analysis of the nature and stability of solutions. Equilibrium solutions in which time derivatives are absent are given by the origin $x=y=z=0$, or (from (1.1a)) $x=y$ whence $z=r-a$ from (1.ib). Eq. (1.1c) thus implies that

$|x|^{2}=b(r-a)$.

Since $z$ is real, it follows that such points can only exist if $\ln (r-a)=0$; that is,

$e+r_{2}=0$

In this case, there is a continuum of steady states given by

$z=r_{1}-1$,

$|x|=|y|=\left[b\left(r_{1}-1\right)\right]^{1 / 2}$.

This rather pathological possibility already reveais the special nature of (1.1). In [1] it was found for the barcclinic two layer model with weak dissipation and weak beta-effect that

$e=3 r_{2}$

$\sigma=2$

The special condition (2.2) is not satisfied and so only the origin is a fixed point. In the case $e=r_{2}=0$ we technically do not return to the full real Lorenz equations as $x$ and $y$ can siill remain complex. The swo fixed points of the real Lorenz equations (in addition to the origin) are replaced by the continuum of points (2.3) although there is very little difference in this intermediate case from the real case.

\subsection{Stability of the origin}

We examine the stability of the steady state $(0,0,0)$ by linearising $(1.1)$ about this point. To do so we simply neglect quadratic terms, thus

$\left(\begin{array}{l}\dot{x} \\ y \\ z\end{array}\right)=\left(\begin{array}{ccc}-\sigma & \sigma & 0 \\ r & -a & 0 \\ 0 & 0 & -n\end{array}\right)\left(\begin{array}{l}x \\ y \\ z\end{array}\right)$.

We note that $z=0$ is always a stable manifold $(\dot{z}=-b z)$. All solutions to (2.6) are proportional to $\exp (\lambda t)$, where the eigenvalues $\lambda$ (assumed distinct) are given by $\lambda=-b$, and $(\sigma+\lambda)(a+$ A) $-r \sigma=0$, whence

$\lambda=\left\lfloor\left[-(\sigma+a) \pm\left\{(\sigma+a)^{2}+4 \sigma(r-a)\right\}^{1 / 2}\right]\right.$. 
If either vaine of $\lambda$ in (2.7) has $\operatorname{Re}(\lambda)>0$, then the origin is said to be linearly unstable. Note thas the two values of $\lambda$ in $(27)$ are not generally complex conjugates; this is hecause the characteristic equation for $\lambda$ does not have real coefficients. If (as can easily be done) the eqs. .1 ) are first written out as a five by five system for the variables

$x_{\mathrm{R}}=\operatorname{Re}(x) ; \quad x_{\mathrm{I}}=\operatorname{In}\left(x ; \quad y_{\mathrm{R}}=\operatorname{Re}(!) ;\right.$

$y_{1}=\operatorname{Im}(y) ; z$

then the resulting matri. equation analogous to (2.6) would have eigenvalues $-b . \lambda$ given by (2.7) together with $\lambda^{*}$.

Let us defint

$p+i q=\left\{(\sigma+a)^{2}+4 \sigma(r-a)\right\}^{1 / 2}: \quad p>0$

(this can be done without loss of generality). Then (2.7) gives the eigenvalues as

$\lambda=\frac{1}{2}[-(\sigma+a) \pm(p+i q)]$

so that

$\operatorname{Re}(\lambda)=\frac{1}{2}[ \pm p-(\sigma+1)]$.

It follows (remember $p>0$ ) that one eigenvalue aiways has negative real part. and the other is negative or positive depending as $p \leqslant c+1$. The critical stability limit is when

$p=a+1$.

and this relation determines a corresponding relation between the parameters which. plotted as a curve in pair: meter space, divides regions of stabiity from those of instability. From (2.8). we find

$p^{2}-q^{2}+2 i p q=(\sigma+a)^{2}+4 \sigma(r-a)$,

whence a little algebra shows that

$$
\begin{aligned}
& p^{2}-q^{2}=(\sigma+1)^{2}+4 \sigma\left(r_{1}-1\right)-e^{2}, \\
& p q=2 \sigma\left(e+r_{3}\right)-e(\sigma+1) .
\end{aligned}
$$

Following Lorenz, we use $r_{1}$ as the bifurcation parameter, and denote its value at ihe stability limit by $r_{1 s}$; thus when $r_{1}=r_{i c} p=\sigma+1$, so (2.12) implies

$e^{2}-q^{2}=4 \sigma\left(r_{1}-1\right)$,
$e+q=\frac{2 \sigma\left(e+r_{2}\right)}{\sigma+1}$.

Note immediately that the frequency $\omega$ of the critically stable eigenmode is given, from (2.9). by

$\omega=\operatorname{lm} \lambda=\{(e+q)$.

Eq. (2.13) implies

$v=\frac{v(e+t)}{v+1}$

We may observe that if $e+r_{2} \neq 0$, then the origin becomes oscillatorily unstable, so that the conditions for a Hopf bifurcation will occur (provided also $\mathrm{d}(\operatorname{Re} \lambda) / \mathrm{d} r_{1} \neq 0$ at $r_{1}=r_{1 \mathrm{c}}$ ): thus we may expect a limit cycle to bifurcate from the origin at $r_{1}=r_{10}$. with approximate frequency $w$ : hence as $e+r_{2} \rightarrow 0$, the frequency tends to zero, and so the continuum of equilibrium points (2.3) may be interpreted as the limit of a limit cycle in which the frequency has decreased to zero. We will generally suppose $\omega \neq 0$.

Eliminating $q$ in (2.13) we obtain

$r_{1:}=1+\frac{\left(e+r_{2}\right)\left(e-\sigma r_{3}\right)}{(\sigma+1)^{2}}$

as the critical value of $r_{1}$. It is easy to see from (2.12) that if $r_{1}<r_{t}$. then $p<\sigma+1$ (and vice versa), so that the origin is linearly stable for $r_{1}<r_{\mathrm{lk}}$, and linearly unstable for $r_{1}>r_{\mathrm{k}}$. 


\subsection{Limit cycle}

From (2.12), it is also easy to check that $\mathrm{d}(\operatorname{Re} \lambda) / \mathrm{d} r_{1}>0$ at $r_{1}=r_{\mathrm{e}}$ so that (provided (I.1) is written as a five by five system, and consideriig the previous remarks about cigenvalues in this case) the Hopf theorem is applicable, and thus a limit cycle does bifurcate from the origin at $r=r_{1 c}$, provided $\omega \neq 0$. Approximate techniques for giving the form of this exist (for $\left.\left|r_{1}-r_{1}\right| \ll 1\right)$. However, numerical computation (fig. 1) shows that even for $r_{1}-r_{\mathrm{lc}} \approx 1$, the limit cycle appears to be an ellipse in the $\left(x_{R}, y_{R}\right)$ plane ( $R$ denoting real part). Also in the $\left(x_{R}, z\right)$ plane it is evident that $z$ quickly reaches constant value.

This suggests that we look for an exact soluticn to the equations in which $z$ is constant, and $x$ and $y$ are sinusoidal. Since (1) is linear in $x$ and $y$ if $z$ is constant, it is clear that such a solution is possible. We put

$$
x=A \mathrm{e}^{\mathrm{i} f t}, \quad y=B \mathrm{e}^{\mathrm{i} f t}, \quad z=M .
$$

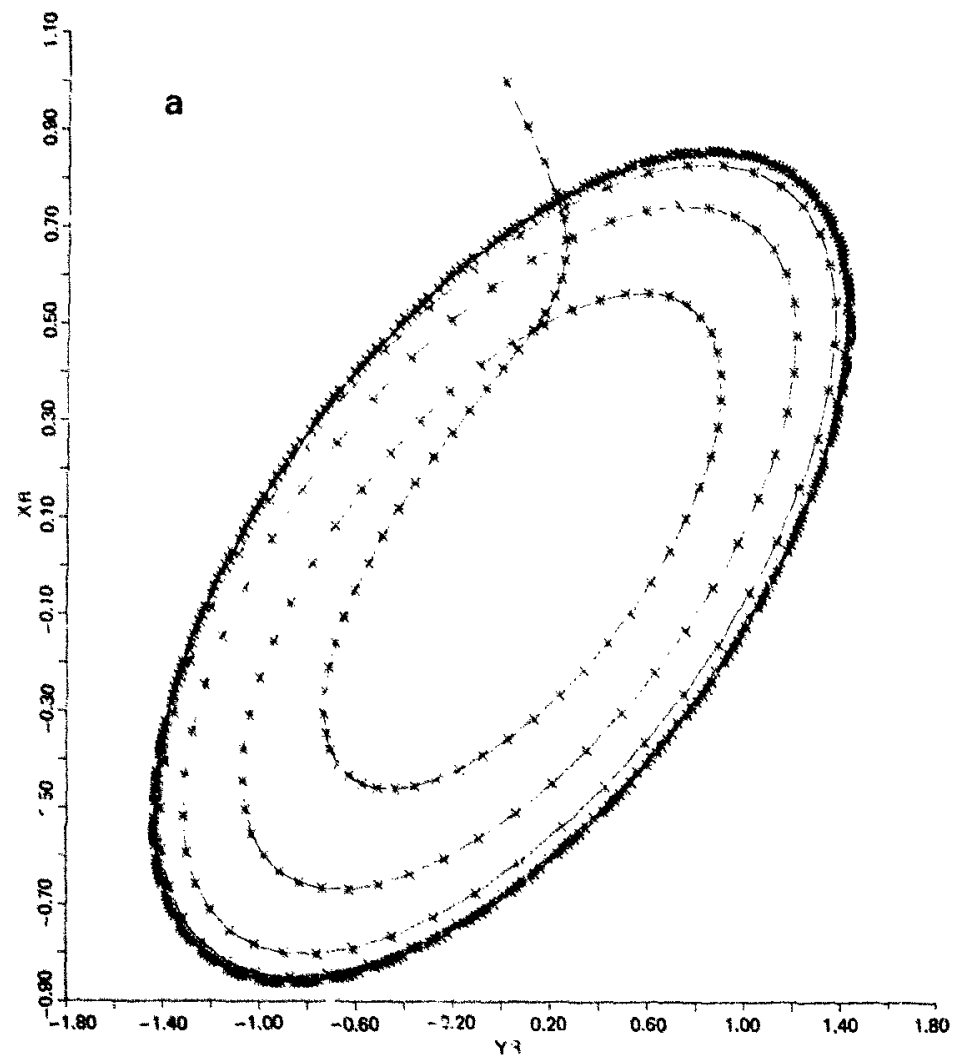

Fig. 1. (a) Stable elliptical limit cycle in the $x_{k-y_{R}}$ plane w th parameter values $b=4 i 3, \sigma=2, n_{1}=2, n_{2}=1, e=3$. 


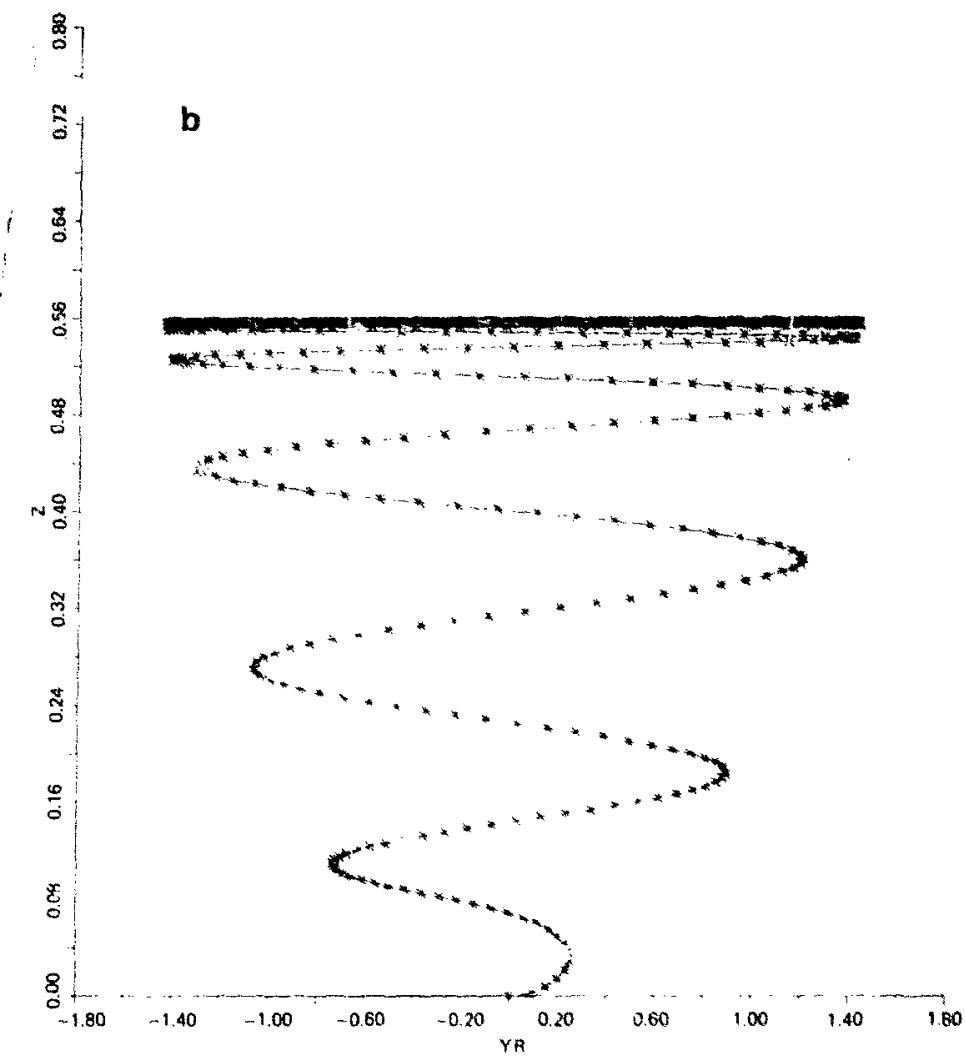

Fig. 1. (b) Plot of $z$ versus $y_{k}$ for the limit cycle in (a) showing z reaching a constant value.

Then (1.1) implies

$(a+i f)\left(1+\frac{i f}{\sigma}\right)=r-|A|^{2} / b$.

if $A=-\sigma A+\sigma B$,

if $B=(r-M) A-a B$.

Equating real and imaginary parts of $(2,19)$

$0=\frac{1}{2}\left(A B^{*}+A^{*} B\right)-b M$, shows that

sol that we find

$f=\omega=\frac{\sigma\left(e+r_{2}\right)}{\sigma+1}$

$\theta=\left(1+\frac{i f}{v}\right) A ; \quad M=|A|^{2} / b$,

and $f$ and $|A|$ are given by

and

$|A|^{2}=b\left(r_{t}-r_{\mathrm{lc}}\right)$ 
(2.20) and (2.21) conform with the dictates of Hopf's theorem, but show that the limit cycle can be determined $2 x a c t l y$ up to a phase factor in $A$. The amplitude increases with $\left(r_{1}-r_{1 k}\right)^{1 / 2}$ so that the bifurcation is supercritical and hence stable (at least initially) and the frequency of the oscillation remains cinnstant. The solution can be written

$x=A \mathrm{e}^{i \omega t} ; \quad y=\left(1+\frac{i \omega}{\sigma}\right) A \mathrm{e}^{i \omega N} ; \quad z=|A|^{2} / b$.

We note that such exact limit cycle solutions may exist for other ordinary differential systems. and in this context we draw attention to the papers of Fujisaka and Yamada [15] and Escher [16].

\subsection{Stability of the limit cycle}

Normally, one cannot explicitly study the stability of a limit cycle, since the basic state is rarely susceptible to analysis. It is therefore very fortunate in the present case that the stability may be explicitly examined, since the limit cycle is exactly known. It is possible to calculate the critical value of $r_{i}$ whers the limit cycle becomes unstable, which we denote by $r_{i}^{\prime}$. This calculation however does not enlighten us as to the qualitative nature of this bifurcation and so this qualitative study is left to section 3 and we confire ourselves to purely calculating $r$ ic*

Firstly we change variabls to those with respect to a rotating frame, which reduces the limit cycle to a fixed point. That is, we put

$x=X \mathrm{e}^{\mathrm{ins} ;} ; \quad y=Y \mathrm{e}^{i \mathrm{iwt}}, \quad z=Z$,

where $\omega$ is given by $(2.20) . X, Y, Z$ satisfy

$\dot{X}=-(\sigma+i \omega) X+\sigma Y$,

$\dot{Y}=(r-Z) X-(a+i \omega) Y$,

$\dot{Z}=\frac{b}{2}\left(X Y^{*}+X^{*} Y\right)-b Z$. which have the fixed points $X=Y=Z=0$ (unstable for $r_{1}>r_{\mathrm{t}}$ ), and also

$X=A: \quad Y=\left(1+\frac{i \omega}{\sigma}\right) A ; \quad Z=|A|^{2} / b$

where $|A|$ is given by (2.21). Thus (2.24) does indeed have a continuum of equilibrium points (each corresponds to the limit cycle), as was the case for (1.1) when $\omega=0$.

We observe that since (2.25) gives an equilibrium point independent of the phase, we may choose without any loss of generality that $A$ is real. Now a steady state solution. of (2.24) exists, in which $A$ is replaced by $A \exp (\mathrm{i} \epsilon)=$ $A+i \epsilon A+O\left(\epsilon^{2}\right)$. It follows that the linearised equations about (2.25) must have a neitrally stable solution ( $\mathrm{i} X_{0}, \mathrm{i} Y_{1,}, 0$ ) (where 0 denotes the steadv state) for all values of $r_{1}>r_{1 c}$. In other words, one eigenvalue of the matrix equation governing such nerturbations will be zero. This physically signifies stability, since it is only a phase shift in the limit cycle, but ensures that the bifurcation does not satisfy the Hopf criterion in its entirety. The nature of this bifurcation and particularly the rôle of the $\lambda=0$ eigenvalue is discussed in more detail in section 3. Furthermore, it is apparent that this discussion should in principle apply to any genuine limit cycle: examination of the present model thus gives an opportunity of explicit comparison of the theory with numerical experiment.

To analyse perturbations about the equilibrium point, we take $A$ real (as above) and set

$$
\begin{aligned}
& X=A+\xi, \\
& Y=\left(1+\frac{i \omega}{\sigma}\right) A+\eta, \\
& Z=A^{2} l b+\zeta .
\end{aligned}
$$

The linearised equations for $\xi, \eta$ and $\zeta$ are then 
$\dot{\xi}=-(\sigma+i \omega) \xi+\sigma \eta$

$\dot{\eta}=\left(r-A^{2} / b\right) \xi-(a+i \omega) \eta-A \xi$.

$\dot{\zeta}=\frac{i}{2} \Lambda\left(1-\frac{i \omega}{\sigma}\right) \xi+\frac{1}{2}\left(1+\frac{i \omega}{\sigma}\right) A \xi^{*}$

$+\frac{1}{2} A \eta+\frac{1}{2} A \eta^{*}-b$.

Because of the precence of $\xi^{*}$ and $\eta^{*}$, these equations carnot imreditely be written in mat-ix form, but must be supplemented by equations for $\xi^{*}$ and $\eta^{2 *}$ :

$\dot{\xi}^{*}=-(\sigma-i \omega) \xi^{*}+\sigma \eta^{*}$,

$\dot{\eta}^{*}=\left(r^{*}-A^{2} / b\right) \xi^{*}-\left(a^{*}-i \omega\right) \eta^{*}-A C$.

We define

$L=a+\mathrm{i} \omega$

$N=1+i \omega / \sigma$.

$P=r-A^{2} b$.

and recalling ( 2.19$)$, we observe that

$L N=P$.

(2.26) and (2.27) may now be written in the form

$\left(\begin{array}{c}\dot{\xi} \\ \eta \\ \xi^{*} \\ \eta^{*} \\ \xi\end{array}\right)=\left(\begin{array}{ccccc}-\sigma N & \sigma & 0 & 0 & 0 \\ p & -L & 0 & 0 & -A \\ 0 & 0 & -\sigma N^{*} & \sigma & 0 \\ 0 & 0 & P^{*} & -L^{*} & -A \\ \frac{1}{2} N^{*} & \frac{1}{2} A & \frac{1}{2} A N^{*} & \frac{1}{2} A & -b\end{array}\right)\left(\begin{array}{c}\xi \\ \eta \\ \xi^{*} \\ \eta^{*} \\ \zeta\end{array}\right)$

and as before, there exist solutions proportional to $\exp (\lambda t)$. At this point we observe that such solutions will be such that $\xi^{*}$ is not the conjugate of $\xi$; this is analogous to having complex exponents $\lambda$ in matrix equations governing real variables. In such cases the linearity of the equations enables suitable superpositions to be chosen so that the variables are neal. Similarly. we would choose a superposition in the present case such that $\xi^{*}$ is the conjugate of $\xi$.

This may be verified by writing (2.30) as a five by five real matrix equation for $x_{R}, y_{R}$, etc. Since the solutions proporitional to $\exp \left(\lambda_{i}^{*}\right)$ give complex conjugate pairs, or real values of $\lambda$, the same must be true of (2.30) (as is shown below). Thus solutions proportional to $\exp (\lambda t)$ exist when $\lambda$ is an eigenvalue of (2.30): that is, when

$$
\begin{array}{ccccc}
-(\sigma N+\lambda) & 0 & 0 & 0 & 0 \\
P & -(L+\lambda) & 0 & 0 & -A \\
0 & 0 & -\left(\sigma N^{*}+\lambda\right) & \sigma & 0 \\
0 & 0 & P^{*} & -\left(L^{*}+\lambda\right) & -A \\
\left\lfloor N^{*}\right. & ! A & \frac{1}{2 A N} & ! A & -(b+\lambda) \\
=0 . & & & & (2.31)
\end{array}
$$

This is casily evaluated by multiplying the elements of the fifth column by its cofactors. We obtain

$$
\begin{aligned}
0= & (b+\lambda)[(\sigma N+\lambda)(L+\lambda)-\sigma P] \\
& \times\left[\left(\sigma N^{*}+\lambda\right)\left(L^{*}+\lambda\right)-\sigma P^{*}\right] \\
& +\left\lfloor A^{2}\left(\sigma N^{*}+\lambda+\sigma N\right)[(\sigma N+\lambda)(L+\lambda)\right. \\
& \left.-\sigma P+\left(\sigma N^{*}+\lambda\right)\left(L^{*}+\lambda\right)-\sigma P^{*}\right] .
\end{aligned}
$$

Using $L N=P$ from (2.29), we find that $\lambda$ is a factor (as predicted): thus $\lambda=0$, or

$$
\begin{aligned}
& \lambda(\lambda+b)[\lambda+(L+N \sigma)]\left[\lambda+\left(L^{*}+N^{*} \sigma\right)\right] \\
& +\frac{1}{2} A^{2}\left(\lambda+\sigma N+\sigma N^{*}\right) \\
& \quad \times\left[(\lambda+L+N \sigma)+\left(\lambda+L^{*}+N^{*} \sigma\right)\right]=0 .
\end{aligned}
$$

which is a quartic polynomial in $\lambda$. We evaluate an explicit stability criterion as follows. Define

$$
\begin{aligned}
& \alpha=(L+\sigma N)\left(L^{*}+\sigma N^{*}\right)=(\sigma+1)^{2}+(2 \omega-e)^{2} \\
& \beta=\left\lfloor\left[L+\sigma N+L^{*}+\sigma N^{*}\right]=\sigma+1,\right. \\
& \gamma=\sigma\left(N+N^{*}\right)=2 \sigma .
\end{aligned}
$$


Eq. (2.33) is thus

$\lambda(\lambda+b)\left(\lambda^{2}+2 \beta \lambda+\alpha\right)+A^{2}(\lambda+\gamma)(\lambda+\beta)=0$.

We have $\alpha, \beta, \gamma>0$; thus the constant term is positive for all $A^{2}>0$. Hence $\lambda=0$ is not a solution of (2.35) and instability can only occur if conjugate roots of $(2.35)$ cross the imaginary axis. For $A^{2}>0, A^{2} \rightarrow 0$, the roots of $(2,35)$ all have negative real part. Therefore the limit cycle is stable for $r_{1}>r_{\text {is }}$ until a critical value $r_{1}=r_{i}^{\prime}$, at which $(2.35)$ has roots $\lambda= \pm i \Omega$. say. At this point (if it exists), (2.35) may be written

$\left(\lambda^{2}+\Omega^{2}\right)\left(\lambda^{2}+p \lambda+q\right)=0$.

Multiplying out (2.35) and (2.36), we find, on identifying coefficients of powers of $\lambda$, that we must have

$p=b+2 \beta$,

$\Omega^{2}+q=\alpha+2 b \beta+A^{2}$.

$p \Omega^{2}=\alpha b+(\beta+\gamma) A^{2}$,

$q \Omega^{2}=\beta \gamma A^{2}$.

Eliminating $p, q$ and $\Omega^{2}$ gives a critical value of $A^{2}$, and hence of $r_{1}$, at which instability sets in. since $\alpha, b, \beta$ and $\gamma$ are all positive, $p, q$, and $\Omega$ are also, and thus (2.36) implies that $\Omega$ is real, and the other two roots of (2.36) have negative real parts. From (2.37), wo have

$$
\Omega^{2}=\left[\alpha b+(\beta+\gamma) A^{2}\right] /(b+2 \beta) .
$$

whence

$q=\frac{\beta \gamma A^{2}(h+z \beta)}{\left[\alpha b+(\beta+\gamma) A^{2}\right]}$

so that

$$
\begin{gathered}
\alpha+2 b \beta+A^{2}=\frac{\alpha b+(\beta+\gamma) A^{2}}{(b+2 \beta)} \\
+\frac{\beta \gamma A^{2}(b+2 \beta)}{\left[a b+(\beta+\gamma) A^{2}\right]}
\end{gathered}
$$

is the stability criterion for $A^{2}$. This is evidentiy a quadratic equation for $A^{2}$,

$Q\left(A^{2}\right) \equiv Q_{1} A^{4}+Q_{2} A^{2}+Q_{3}=0$,

where some simplification shows that

$$
\begin{aligned}
Q_{1}= & (\beta+\gamma)(\gamma-b-\beta) \\
= & (3 \sigma+1)(\alpha-b-1), \\
Q_{2}= & \beta(b+2 \beta)(2 \beta \gamma-2 b \beta-b \gamma) \\
& -\alpha\left(-b \gamma+b^{2}+\beta b+2 \beta^{2}+2 \beta \gamma\right), \\
Q_{3}= & -2 \alpha \beta b\left[\alpha+b^{2}+2 \beta b\right] .
\end{aligned}
$$

We have $Q_{3}<0$. If $a>b+1$, then $Q_{1}>0$ and (2.41) has a unique positive root, which determines the critical value of $A^{2}$. This turns : $t$ to be

$\rho=\frac{-Q_{2}+\left[Q_{2}^{2}-4 Q_{1} Q_{3}\right]^{1 / 2}}{2 Q_{1}}$.

In this case the critical value of $r_{1}$ where the limit cycle becomes unstable is given by

$r_{i c}^{\prime}=r_{i c}+\rho / b$,

where $\rho$ is given by (2.43), $Q_{1}, Q_{2}$ and $Q_{3}$ by (2.42), $\alpha, \beta$ and $\gamma$ by (2.34) and $\omega$ by (2.20). This is a useable, though messy criterion.

If $\sigma<b+1$, then $Q_{1}<0$ and $Q$ may attain positive values for a finite range of $A^{2}$. A necessary condition that this occurs is that $Q_{2}>$ 0 . Using the definitions of $\beta$ and $\gamma$ in 2.34), together with the fact that $\alpha \geq \beta^{2}$ and $b>\sigma-1$, evaluation of $Q_{2}$ in (2.42) shows that necessarily $Q_{2}<0$. It follows that the limit cycle is always stable if $\sigma<b+1$, which is identical to the corresponding real case.

To summarise, we have shown that the limit cycle, in the form of the exact solution (2.22), is always stable if $\sigma<b+1$. If $\sigma>b+1$ then it is also stable for values of $r_{1}$ in the range $r_{i c}<r_{1}<$ $r_{\mathrm{ic}}$ but becomes unstable when $r_{1}>r_{1 \mathrm{c}}^{\prime}$. Due to the presence of the zero eigenvalue of the linearised matrix equation the bifurcation is not 
completely ri Hopf type although without this eigenvalue a Hopf bifurcation would occur The nature of his bifurcation thus requires further consideration.

3. Hifurcation of the limit cycle in the complex Lor $\geqslant$ eyuations

\subsection{General case}

Let us first cons der the gescral case of bifurcation of a timit cycle. Siraightforwad linearised perturbation analysis may be carried out, leading to a linea $m_{\text {s. }}$ rix equation for the perturbations in which the matrix is periodic of period $T$. say (where $T$ is the period of the underlying limit cycle). Floquet theory (see e.g., Coddington and Levinson (7]) then tells us that the solutions of this linear equation may be written as the product of a function of period $T$ and the function $\exp (\mu t)$. Stability then rests on the magnitude of the Floquet multipliers $\exp (\mu 7)$, and thus on the nature of the Floquet exponents $\mu$. In the case of our analysis of the complex Lorenz limit cycle, the exponents $\mu$ are essentially the same as the eigenvalues $\lambda$.

Various possibilities for bifurcation now occur, and are discussed by Ruelie and Takens [8. Joseph [9] and Lanford [10]. Particularly, if $A$ crosses the imaginary axis at i $\Omega$ (and so also $\lambda^{*}$ at $-i \Omega$ ), then the limit cycle bifurcates to motio: on a 2-torus. Generally this motion will be doubly periodic (corresponding to a "Hopf bifurcation" in a frame rotating with the limit cycle. or of the associated Poincare map): for particular values of $A$, subharmonic periodic solutions may also occur (Joseph [9]).

However, Floquet theory is not generally useful for explicit calculations, and in addition the limit sycle solution is not usually known explicitly. Thus practical applications of such ifreorems as exist do not seem prevalent in the "terature: particularly, we are unaware of a method for computation of the stability of the bifurcating torus. In the Hopf case, there are numerous "different" methods which can be used, which probably amount to the same idea: the center manifold theorem. Hopt's theorem. the Poincare-Lindstedt method, the KrylovBogoliubov-Mitropolsky method of averaging. and the Cole-Kevorkian method of multiple scales, as originally developed for fluid fows by Stuart [11]. The last-named method derives an amplitude equation for marginally stable oscillatory perturbations of the form

$x=x_{0}+\left[\epsilon A(\bar{t}) \mathrm{e}^{\mathrm{in} t} u_{n}+(*)\right]+\cdots$,

wherein $\epsilon$ is a measure of the amplitude $A: i=$ $\epsilon^{\prime} t$ is a slow time vaiable, and $\Omega$ is the marginal frequency. Uniformly valid expansions of the form (3.1) require $A$ to satisfy an equation of the form

$\frac{\mathrm{d} A}{\mathrm{~d} t}=k_{1} A+k_{2} A|A|^{2}$

a result obtained by McLaughlin and Martin [6] in their classification of the bifurcations in the real Lorenz model. Along with the various different methods mentioned above. our cal. culation is essentially equivalent to theirs.

If $\operatorname{Re} k_{2}>0$, then the bifurcation is subcritical, and the limit cycle is unstable; if $\operatorname{Re} k_{2}<0$, the bifurcation is supercritical and stable. Calculation of $k_{2}$ is straightforward but messy.

The distinction between super- and s'b-critical bifurcations is an important one in the context of urbulence and chatic trajectories of differential equations. Ruelle and Takens [8] proposed that the trajectories on higher dimensional tori (corresponding to further bifurcations of the system under consideration) would not generally be of periodic or almost periodic type; rather, they could approach "strange" attractors. which for all practical purposes would appear turbulent, or chaotic. It is clear that this scheme is only viable if the intermediate bifurcations are supercritical, so that the trajectories 
on the various tori are stable (in that they approach the torus). It is for this reason that it is useful to analyse the direction of bifurcation in real cases. In fluid fow along pipes, bifurcation (when it occurs) is of subcritical type, and so the Ruelle-Takens ideas ars presumably irrelevant. In the Benard problem, the first bifurcation, and in the Taylor column, the first two bifurcations are supercritical and stable, and tirbulence in these cases may be along the lines of the abstract theory.

For the above reasons, we wish to examine the direction of bifurcation of the 2-torus for the complex Lorenz equations. Our approach will be constructive; that is, we will use the formal method of multiple scales to find amplitude equations satisfied by the perturbations of the limit cycle. An analysis of these gives conditions of stability and direction of bifurcation of the 2-torts. Since the bifurcation 's subcritical in the real case (M/cLaughlin an I Martin [6]), we expect this to be also true in the present case. at least for sufficiently small $e$ and $r_{2}$.

\subsection{Rotationally ineariant systems}

The procedure we adopt is straightforward. A similar general method is given by Haken [12], who follows somewhat the apprnach of Eckhaus [13]. This determines an infinity of amplitude functions, of which only a finite set are relevant: an unstable mode and a set of "slaved" modes, in Haken's terminolugy. We prefer to adopt the method of multiple scales (e.g. Nayfeh [14]), since then the approximate expansions adopted are made explicit from the outset, and the naturs of the equations is then apparent.

Let us consider the system of real-valued ordinary differential equations

$\frac{d x_{i}}{d t}=f_{i}(x ; \mu)$.

We shall suppose that this system is derivea via a change of variables from another system, in which a limit cycle exists for a certain range of $u$ : we shall refer to this as the underlying limit cycle. For the complex Lorenz equations, we obtain (3.3) via the change of variables $(x, y, z) \rightarrow\left(x \mathrm{e}^{\mathrm{i} \omega t}, y \mathrm{e}^{\mathrm{i} \omega t}, z\right)$ : generally, we can change into a rotating frame in this way for any system with an underlying limit cycle, but we expect to obtain a non-autonomous system unless the oscillation is exactly sinusoidal In this case, it is reasonaible to adopt the following assumptions. Let $x_{i j}$ be a fixed point of (3.3) corresponding to an underlying limit cycle. We define a rotation matrix $R(t)$ which satisfies $R(\alpha) R(\beta)=R(\alpha+\beta)$, and the underlying limit cycle is given by $R(t) x_{0}$ (i.e.. $(3,3)$ is obtained from the original system via the change of variables $x \rightarrow R x$ ). Since then we have that

$f\left[R x_{0} ; \mu\right]=0 . \quad R=R(\alpha)$.

for all $\mu$ anc $\alpha$. (i.e., (3.3) has a continuum of equilibria), it is reasonable to assume that (3.3) is invariant under rotation; that is, if $y=R(\alpha) x$, then $\mathrm{d} y / \mathrm{d} t=f(y ; \mu)$. This implies that $f$ satisfies

$R f(x ; \mu)=f(R x ; \mu)$

for all $x$ and $\alpha$. This condition is satisfied by the complex Lorenz equations, for example (see below). Differentiation of (3.5) with respect to $\alpha$ yields

$R_{i j}^{\prime}(\alpha) f_{j}(x ; \mu)=f_{i, j}(R x ; \mu) R_{j k}^{\prime}(\alpha) x_{k} ;$

putting $x=x_{0}$ immediately shows, using (3.5), that

$f_{i, j}\left(R x_{0} ; \mu\right) R_{j k}^{j} x_{0 k}=0$.

In other words the Jacobian matrix $D f=$ $\left(f_{i, j}\left(R x_{0} ; \mu\right)\right)$ has, for every value of $\mu$ (and $\alpha$ ), a right eigenvector $u_{0}$ with corresponding eigenvalue zero, where $u_{0}$ is given by

$\boldsymbol{u}_{0}=\mathbf{R}^{\prime}(\alpha) \boldsymbol{x}_{\mathbf{0}}$ 
Since linear perturbations to $\boldsymbol{x}_{0}$ are proportionai to $\exp (\lambda t)$, where $\lambda$ is an eigenvalue of $D f$. it follows that $x_{0}$ is (at least) ncutrally stable for all $\mu$. However, this is by virtue of the fact that the undr rlying limit cycle may have its phase perfurted, but yet be arbitrarily stable; thus we discount $\lambda=0$ as represening a tate of marginal stability, since if all other $\operatorname{Re} \lambda$ are less than zero, then the underlying imit cycle is structurally stable.

If we differentiate 3.5 ) with respect to $x$. we find

$R(D f(x ; f))=(D f(R x: \mu)) R$.

Now suppose that $U$ is a right eigenvector of $D f(x ; \mu)$ with eigenvalue $A:$ then pre-multiplying $U$ by (3.8a) show's that $R U$ is the corresponding eigenvector of $D f(R x ; \mu)$. The use of this is that $U$ is independent of $\alpha$ in $R(\alpha)$. Particularly, (3.8) implies (with an obvious notation)

$U_{0}=R^{\prime}(0) x_{0}$

whence $u_{0}=R(\alpha) R^{\prime}(0) x_{0}$, which is consistent with (3.8), sivice consideration of $R(\alpha+\beta)=$ $R(\alpha) R(\beta)$ shows that $R^{\prime}(\alpha)=R(\alpha) R^{\prime}(0)=$ $R^{\prime}(0) R(\alpha)$.

Let us denote $f_{i, j}\left(x_{0} ; \mu\right)$ by $f_{i, j}^{0}$ : we will now assume that (apart from the zero eigerivalue), all other eigenvalues of $f_{i, j}^{0}$ are such that $\operatorname{Re}(\lambda)<0$. but that at $\mu=\mu_{0}$. a pair of eigenvalues \pm i . $\Omega>0$, cross the imaginary axis in such a way that $\mathrm{d}(\operatorname{Re} \lambda) / \mathrm{d} \mu \mid \mu_{\mathrm{c}}>0$ : the corresponding (critical) eigenveciors of $f_{i, j}^{0}$ are $U_{n}$ and $U_{3}^{*}$. These conditions (apart from the zero eigenvalue) resemble those of the Hopf bifurcation, and are valid in the complex Lorenz case. From the paragraph above, it follows that $f_{i, j}\left(R x_{0} ; \mu\right)$ has - vrresponding eigenvectors $u_{\Omega}=R U_{\Omega}$ and $u_{\Omega}^{*}=$ $R U_{0}$ with eizenvalues $+i \Omega$ and $-i \Omega$, respestrely.

Wo now seek an approximate solution when ' $\mu-\mu_{c} \mid \ll 1$; accordingly we den̂ne (with some foresight)

$\mu=\mu_{i} \pm \epsilon^{2}, \quad 0<\epsilon \ll 1$.

where the plus and minus signs refer respectively to super- and sub-critical states (weakly unstable and weakly stable). We also define the slow time scale

$\tau=\epsilon^{2} t$

and will seek solutions to (3.3) in the form

$x=x^{(0)}+\epsilon x^{(1)}+\epsilon^{2} x^{(2)}+\cdots$

where $x^{(i)}=x^{(i)}(t, \tau)$. The procedure as usual is straightforward, but there are one or two subleties which distinguish the expansion from the more conventional Hopf case. Substituting (3.9), (3.10) and (3.11) ir.to (3.3), we obtain

$$
\begin{aligned}
& \left(\frac{\partial}{\partial t}+\epsilon^{2} \frac{\partial}{\partial t}\right)\left(x^{(0)}+\epsilon x^{(1)}+\epsilon^{2} x^{(2)}+\epsilon^{3} x^{(3)}+\cdots\right) \\
& =f\left[x^{(0)}+\epsilon x^{(1)}+\cdots ; \mu_{i} \pm \epsilon^{2}\right] .
\end{aligned}
$$

whence we derive

$$
\begin{aligned}
& {\left[\frac{\partial x_{i}^{(0)}}{\partial t}+\epsilon \frac{\partial x_{i}^{(n)}}{\partial t}+\epsilon^{2} \frac{\partial x_{i}^{(2)}}{\partial t}+\epsilon^{3} \frac{\partial x_{1}^{(3)}}{\partial t} \cdots\right]} \\
& +\left[\varepsilon^{2} \frac{\partial x_{i}^{(0)}}{\partial \tau}+\epsilon^{3} \frac{\partial x_{i}^{(1)}}{\partial \tau}+\cdots\right] \\
& =\left[f_{i}+\left\{\epsilon x^{(1)}+\epsilon^{2} x_{i}^{(2)}+\epsilon^{3} x_{j}^{(3)} \cdots\right\} f_{i, j}\right. \\
& \left.+\left\{\langle\epsilon x\}^{\prime \prime}+\epsilon^{2} x_{i}^{2} \cdots\right\}\left\langle\epsilon x_{k}^{(1)}+\epsilon^{2} x\right\}^{2} \cdots\right\} f_{i, j k}^{c}
\end{aligned}
$$

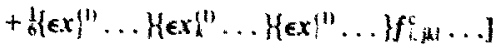

$$
\begin{aligned}
& \pm \epsilon^{2}\left[\frac{\partial f_{i}^{i}}{\partial \mu}+\left\{\boldsymbol{x x}_{i}^{\prime \prime \prime} \ldots\right\} \frac{\partial f_{i j}^{c_{j}}}{\partial \mu}+\cdots\right] \ldots
\end{aligned}
$$

where $f_{i, j}$ denotes $f_{i,}$ evaluated at $x^{(B)}$ and $\mu_{c}$. etc. Equating terms of $O(1)$, we have

$\frac{\partial x_{i}^{(0)}}{\partial t}=f_{i}\left(x^{(0)} ; \mu\right)$ 
The relevant $t$-independent solution of $(3.14)$ is given, from (3.4), by

$x^{(0)}=R(\phi) x_{0}$,

where $\phi=\phi(\tau)$ is a slowly-varying function of time; essentially, it is the phase shift in the underlying limit cycle. At $O(\epsilon)$, we have the linearised equation

$\frac{\partial x_{i}^{(1)}}{\partial t}-f_{i, j}^{c} x_{i}^{(1)}=0 . \quad f_{i, j}^{\prime}=f_{i, j}\left[R(\phi) x_{0} ; \mu_{\mathrm{c}}\right]$

Neglecting initial transients, this equation has solutions proportional to $u_{0}, u_{\Omega} \mathrm{e}^{\mathrm{i} \Omega}$ and $u_{n}^{*} \mathrm{e}^{-\mathrm{int}}$; however, notice that if, in (3.15) $\phi=$ $\phi_{0}+\epsilon \phi_{1}+\cdots$, then

$x^{(\theta)}=R\left(\phi_{0}\right) x_{0}+\epsilon R^{\prime}\left(\phi_{0}\right) x_{0} \phi_{1}+\cdots$

Since $\mu_{0}=R^{\prime}\left(\phi_{0}\right) x_{0}$, it is clear that we may absorb the term propertional to $u_{0}$ in $x^{(1)}$ into $\phi$. In fact we can do this at each stage of the solution. provided we let $\phi$ depend on $\epsilon$ as weil as $t$. Then the solution of (3.16), neglecting transients, may be written

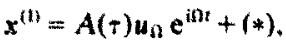

where $(*)$ denotes the complex conjugate.

At $\sigma\left(\epsilon^{2}\right)$. we have, since $\partial f_{i}^{i} / \partial \mu=0$ (from (3.4)),

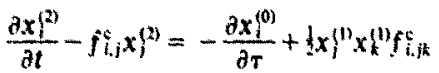

$$
\begin{aligned}
& =-R_{i j}(\phi) \frac{\mathrm{d} \phi}{\mathrm{d} \gamma} x_{0 j}+f_{i_{i k}}^{\mathrm{c}}|A|^{2} u_{n j} u_{n k}^{*} \\
& +\left[\frac{1}{f} f_{i k} A^{2} e^{2 i n t} u_{n j} u_{n k}+(*)\right] \text {, }
\end{aligned}
$$

since $f_{i, j}=f_{i, \mathrm{kj} .}$. The term in $\mathrm{e}^{\mathrm{int}}$ gives a particular solution proportional to $\mathrm{e}^{\text {zint}}$; however (since zero is an eigenvalue of $f_{i, j}$ ) the constant terms may be secular, and it is the elimination of these secular terms which determines the phase $\phi(r)$. Specifically, if a (complex-valued) matrix $A$ has an eigenvalue zero, then the equation $A x=c$ has a solution if and only if $\tilde{v}^{T} c=0$, where a bar denotes the complex conjugate, for all $v$ such that $\bar{A}^{T} v=0$. Thus if $\lambda$ is an eigenvalue of $D f=\left(f_{i, j}^{c}\right)$, then the equation

$\frac{\partial x}{\partial t}-(D f) x=c \mathrm{e}^{\lambda t}$

has a solution proportional to $\mathrm{e}^{\lambda t}$ if and only if $v^{T} \mathrm{c}=0$ where $[\overline{(D f)-\lambda l}]^{T} \bar{v}=0$ : i.e. $(D f)^{T} v^{*}=$ $A^{*} v^{*}$ (* denotes complex conjugate) since $D f$ is real. Now let $v_{0}$ and $v_{n}$ be the left eigenvectors of $D f$. corresponding to the eigenvalues zero and i $\Omega$ respectively, thus

$v_{0}^{T}\left(D_{j}\right)=0$, i.e. $v_{i n f_{i, j}^{c}}^{c}=0$,

$v_{\Omega}^{T}(D f)=\mathrm{i} \Omega v_{\Omega}^{T}$. i.e. $v_{\Omega} f_{\mathrm{i}, \mathrm{i}}^{\mathrm{c}}=\mathrm{i} \Omega v_{\Omega i}$;

then $(D f)^{T} v^{*}=0,(D f)^{T} v *=-i \Omega v *$, and thus it follows that the equation

$\frac{\partial x}{\partial t}-(D f) x=c_{0}+c_{n} \mathrm{e}^{\mathrm{i} \Omega t}$

can only have a solution of the form $a_{0}+a_{\Omega} \mathrm{e}^{\mathrm{i} \Omega t}$ if the constraints

$v_{0} \cdot c_{0}=v_{\Omega} \cdot c_{\Omega}=0$

are satisficd: otherwise, secular terms $t, t \mathrm{e}^{\mathrm{i} \Omega t}$ will occur. Applying this to (3.18), we require $\phi$ to satisfy, recalling (3.8),

$\frac{\mathrm{d} \phi}{\mathrm{d} \tau}=\left[\frac{v_{0 i} f_{i, i \mathrm{i}}^{\mathrm{c}} u_{0 j} u_{i k}^{*}}{u_{0 i} v_{0 i}}\right]|A|^{2}$.

If (3.23) is satisfied, then a paricular solution of (3.18) can be written

$x^{(2)}=a \delta^{(2)}|A|^{2}+\left[a 2_{2}^{(2)} A^{2} e^{2 i n t}+(*)\right]$,

where

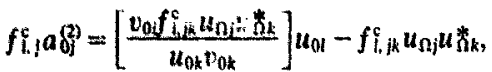


and

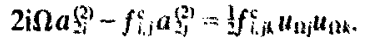

We have already decided to incorporate cons' int solutions proportional to $u_{0}$ into $\phi$; sumilarly, there is no loss in generality in ab. sorbing the solutions proportional to $u_{n} e^{\text {ilt }}$ into $A(\tau)$, provided atsn we unders'and $t=A(\tau ; \epsilon)$. Then (3.24) is the complete solution at $O\left(\epsilon^{3}\right)$, and we turn to $\left(; y^{3}\right)$ terms. Tr:ase are

$$
\begin{aligned}
& \frac{\partial x_{i}^{(3)}}{\partial \hat{i}}-f_{i, j}^{\mathrm{c}} x^{(3)}=-\frac{\partial x_{i}^{(*}}{\partial \tau}+\left\{\left\{x_{i}^{(1)} x_{k}^{(2)}+x_{i}^{(3)} x_{k}^{(1)}\right\} f_{i, j k}^{c}\right. \\
& \left.+\frac{1}{6} x\right)^{(1)} x\left(\left.x^{(1)} x\right|^{(1)} f_{i, j, i}^{c} \pm x\right)^{(1)} \frac{\partial f_{i, j}^{c}}{\partial \mu} .
\end{aligned}
$$

and we again have to eliminate terms on the right-hand side which are secular. From (3.17) and $\mathbf{3 . 2 4}$ ), it is apparent that there are no constant secular terms, and thus we only need choose $A$ such that the $\mathrm{e}^{\mathrm{i} m}$ terms are nonsecular; we in fact obtain the Landau-Stuart equation (3.2), as previously discussed. The coefficients are determined as follows. The coefficient of $\mathrm{e}^{\mathrm{i} / \mathrm{t}}$ in (3.27) is, recalling that $u_{41}=$ $R(\phi) U_{12}$ and that $R^{\prime}(\phi)=R^{\prime}(0) R(\phi)$,

$$
\begin{aligned}
& \frac{\mathrm{d} A}{\mathrm{~d} \tau} u_{n j} \pm A \frac{\partial f_{i j}^{c}}{\partial \mu} u_{n j}-A R_{i j}^{\prime}(0) u_{n j} \frac{\mathrm{d} \phi}{\mathrm{d} \tau}
\end{aligned}
$$

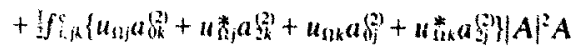

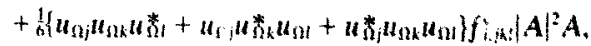

so it follows from (3.22), using (3.23), that the Landau equation for $A$ is

$$
\frac{\mathrm{d} A}{\mathrm{~d} \tau}= \pm k_{1} A+k_{2}|A|^{2} A
$$

whore

$$
k_{1}=v_{n i} \frac{\partial f_{i}^{c} i}{\partial \mu} u_{n j} / u_{n i} v_{n i}
$$

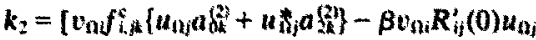

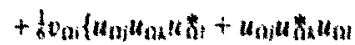

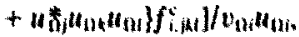

$$
\begin{aligned}
& \beta=\frac{t_{0 L} u_{i, k} u_{1} u_{k_{k}}}{k_{n} v_{0 i}}
\end{aligned}
$$

$k_{\mathrm{I}}=\mathrm{d} \lambda /\left.\mathrm{d} \mu\right|_{\mu=\mu_{\mathrm{c}}}$ is the linear growth rate (Re $k_{1}>0$ by assumption). Therefore, the bifurcation is supercritical if $\operatorname{Re} k_{2}<0$, and subcritical if $\operatorname{Re} k_{2}>0$ since $\left.|A|\right|^{2}$ satisfies

$\frac{\mathrm{d}|A|^{2}}{\mathrm{~d} \tau}= \pm 2\left(\operatorname{Re} k_{1}\right)|A|^{2}+2\left(\operatorname{Re} k_{2}\right)|A|^{4}$

Thus the stability of the bifurcating solutions is determined in exactly the sume manner as in a Hopf bifurcation. It is not obvious (though we might suspect) that $\beta, k_{1}$ and $k_{2}$ are independent of $\tau$, since $u_{1}, u_{0}, v_{n}$ and $v_{0}$ are functions of $\tau$. however. using $u_{n}=R U_{n}, u_{0}=R t_{0}$, and the equivalent formulae for $v_{n}$ and $v_{0}$, it is not difficult to check, using $x$-differentials of $(3.5)$. that this is indeed the case. For example. premultiplication of (3.8a) by $v$. a left eigenvector of $D f(R x: \mu)$. easily shows (if $V$ is the corresponding eigenvector of $D f(x ; \mu)$ ) that $V^{T}=$ $v^{\top} R$. It easily follows that we have $v_{0}^{T}=V_{0}^{\top} R^{-1}$, $v_{n}^{r}=V_{n}^{T} R^{-1}$. We then have $v_{0 i} u_{0 i}=v_{0}^{\top} u_{0}=$ $V_{0}^{T} R^{-1} R U_{0}=V_{0}^{1} U_{0}$, which is independent of $\phi$. and hence of $\tau$. Similar considerations show that $\beta, k_{1}$ and $k_{2}$ are all independent of $\tau$.

\subsection{Nature of the solutions}

The solution can be written as

$$
x=R[\phi(\tau)] x_{0}+\epsilon\left[A(\tau) u_{n} e^{i \Omega t}+(*)\right]+O\left(\epsilon^{2}\right),
$$

where

$\frac{\mathrm{d} \phi}{\mathrm{d} \tau}=\beta|A|^{2} . \quad \frac{\mathrm{d} A}{\mathrm{~d} \tau}=k_{1} A+k_{2}|A|^{2} A$.

If the bifurcation is supercritical, then for $\operatorname{Re} k_{1}>0$, i.e., $\mu>\mu_{\text {c }}$, the solution for $x$ tends to 
a doubly periodic motion in the rotating plane. This consists of a fast $(t \sim 1)$ oscillation of amplitude order $\sqrt{\mu-\mu_{i}}$ about the equilibrium point, which itself precesses periodically around the underlying limit cycle. In the rotating plane. time-plots of $x_{i}$ versus time will thus consist of a fast oscillation whicis (if $u_{0 i} \neq 0$ ) is superimposed by a larger scale slowly oscillating solution. This is observed in the complex Lorenz equations (see fig. 2).

In the fixed frame, we have $x_{F}=R x$, therefore (3.32) is, using the property that $R(\alpha) R(\beta)$ $=R(\alpha+\beta)$, and that $u_{n}=R U_{n}$.

$x_{1}=R(t+\phi(\tau))\left[x_{0}+\epsilon\left\{A(\tau) t_{n} e^{i n t}+(*)\right\}+\phi\left(\epsilon^{\prime}\right)\right\}$

In the limit as $r \rightarrow \infty$ (with $\left.\operatorname{Re} k_{2}<0, \operatorname{Re} k_{1}>0\right)$ ). we have

$|A|^{2} \rightarrow-\left(\frac{\operatorname{Re} k_{1}}{\operatorname{Re} k_{2}}\right)=-\frac{k_{1 R}}{k_{2 R}}$ thus

$$
\begin{aligned}
\phi & =-\frac{\beta k_{1 R}}{k_{2 R}} \tau \\
A & \sim \exp \left[\left(k_{1}-k_{2} \frac{k_{1 R}}{k_{2 R}}\right) \tau\right] \\
& =\exp \left[\mathrm{i}\left\{\frac{k_{11} k_{2 R}-k_{21} k_{1 \mathrm{R}}}{k_{2 \mathrm{R}}}\right\} \tau\right] .
\end{aligned}
$$

It follows that the solutions in the fixed frame can be written in somponent form as the sum of products of two functions of distinct periods, thus

$$
\begin{aligned}
x_{\mathrm{F} i}= & R_{i j}[t+\phi(\tau)] x_{0 j} \\
& +\epsilon\left\{A(\tau) R_{\mathrm{ii}}(t+\phi(\tau)) U_{0 ;} \mathrm{e}^{\mathrm{i} I I t}+(*)\right\}+\mathscr{O}\left(\epsilon^{2}\right) \\
= & \sum_{i} p_{j}^{(i)}(t) q_{j}(t) .
\end{aligned}
$$

where

$$
\begin{aligned}
& p f^{(i)}(t)=R_{i j}(t+\phi(\tau)), \\
& q_{j}(t)=x_{0 j}+\epsilon\left\{A(\tau) U_{0 j} \mathrm{e}^{i n t}+(*)\right\}+\sigma\left(\epsilon^{2}\right),
\end{aligned}
$$
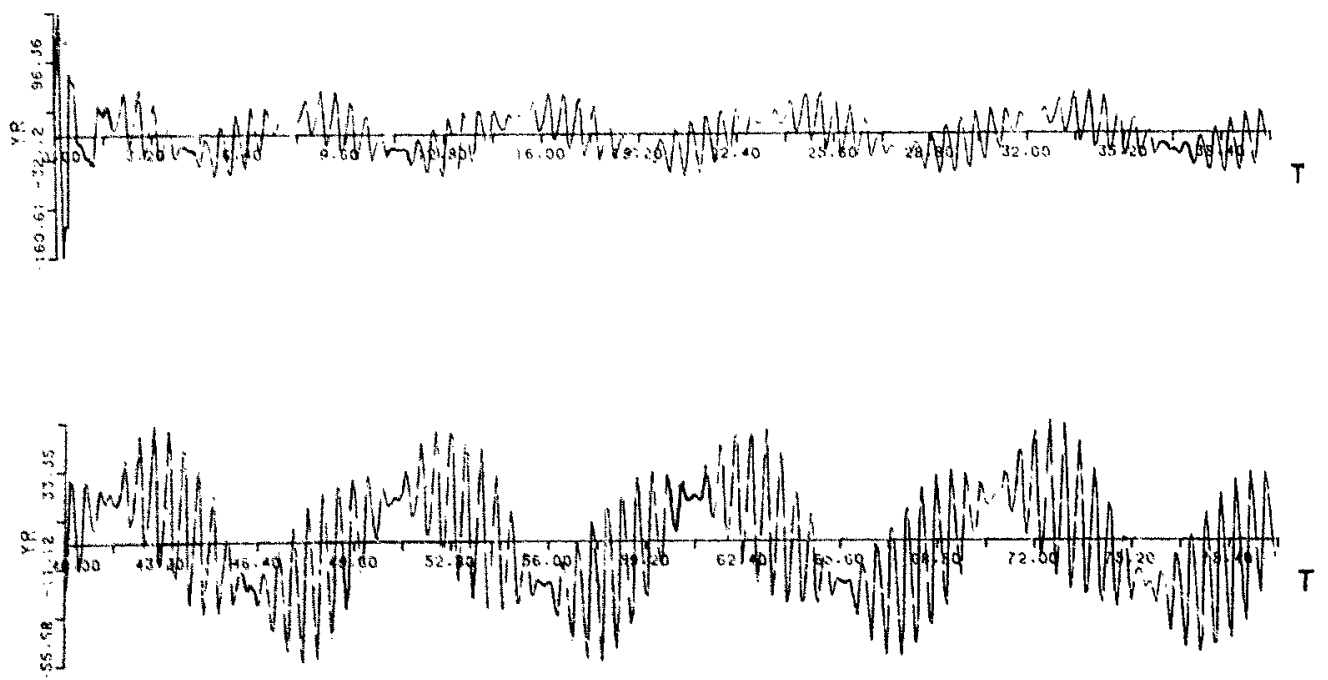

Fig. 2. Plot of $y_{k}$ versus time at $b=0,8, a=2, n \neq 220, n_{z}=1$ and $e=3 r_{3}$, at which there is a stable finite anplitude motion. Note the slow and fast pertiods in the oscillations. 
and $p i t$ and $q_{i}$ have periods $\omega_{1}$ and $\omega_{2}$ respectively. we get

where

$$
\begin{aligned}
& \omega_{1}=\omega\left[1-\epsilon^{2} \frac{\beta k_{1 \mathbb{R}}}{k_{2 \mathbb{R}}} \cdots\right], \\
& \omega_{2}=\Omega+\epsilon^{2}\left\{\frac{k_{11} k_{3 R}-k_{3 \mathrm{~g}} k_{1 \mathrm{R}}}{l_{\mathrm{ZR}}}\right\} \cdots
\end{aligned}
$$

The presence ot two distinct, generally incominensurate frequen.ies characterise these solutions as doubly periodic. A spectrai analysis of the selutions should thus reveal a peak at the fundamental frequency $\omega_{1}$, together with smaller peaks, at $\left|\omega_{1} \pm \omega_{2}\right|$. etc. Such behaviour is found by Fenstermacher. Swinney and Gollub [17] in an experimental analysis of Taylor vortex flow.

\subsection{Application to complex Lorenz equations}

In the rotating plane, the complex Lorenz equations are

$X=-(\sigma+\mathrm{i} \omega) X+\sigma Y$.

$\dot{\gamma}=(r-Z) X-(a+i \omega) Y$.

$\dot{Z}=\frac{1}{2}\left[X Y^{*}+X^{*} Y\right]-b Z$.

whinto-

$\alpha=\frac{\sigma(e+r)}{\sigma+1}$

The non-zero equilitria are

$X=A . \quad Y=A\left(1+\frac{i \omega}{\sigma}\right), \quad Z=|A|^{2} / b$,

$|A|^{*}=b\left(r_{1}-r_{1}\right)$

$r_{i:}=1+\frac{\left(e+r_{2}\right)\left(e-\sigma r_{2}\right)}{(\sigma+1)^{2}}$.

Note that the equations are invariant under the rotation $(X, Y, Z) \rightarrow(X \exp (i \alpha), Y \exp (i \alpha), Z)$. Putting

$X=A\left(x_{1}+i x_{2}\right), \quad Y=A\left(x_{3}+i x_{4}\right), \quad Z=\frac{|A|^{2}}{b} x_{5}$,

$$
\begin{aligned}
\dot{x}_{1}+i \dot{x}_{2}=-(\sigma+i \omega)\left(x_{1}+i x_{2}\right)+\sigma\left(x_{3}+i x_{4}\right), \\
\dot{x}_{3}+i \dot{x}_{4}=\left[r_{1}+i r_{2}-\left(r_{1}-r_{1 k}\right) x_{5}\right]\left(x_{1}+i x_{2}\right) \\
-(1-i e+i \omega)\left(x_{3}+i x_{4}\right) . \\
\dot{x}_{5}=b\left[\left\{\left(x_{1}+i x_{2}\right)\left(x_{3}-i x_{4}\right)\right.\right. \\
\left.\left.+\left(x_{1}-i x_{2}\right)\left(x_{3}+i x_{4}\right)\right\}-x_{5}\right] .
\end{aligned}
$$

whence

$\dot{x}_{1}=-\sigma x_{1}+\omega x_{2}+\sigma x_{3}$

$\dot{x}_{2}=-\omega x_{1}+\sigma x_{2}+\sigma x_{4}$

$\dot{x}_{3}=\left[r_{1}-\left(r_{1}-r_{4}\right) x_{5}\right] x_{1}-r_{2} x_{2}-x_{3}+(\omega-e) x_{4}$,

$\dot{x}_{4}=r_{2} x_{1}+\left[r_{1}-\left(r_{1}-r_{14}\right) x_{4}\right] x_{2}-(\omega-e) x_{3}-x_{4}$.

$\dot{x}_{5}=b\left[x_{1} x_{3}+x_{2} x_{4}-x_{5}\right]$.

with an equilibrium point

$x_{1}=1, \quad x_{2}=0, \quad x_{3}=1 . \quad x_{4}=\frac{\omega}{\sigma}, \quad x_{5}=1$.

By rotation through an angle $\alpha$ of $X$ and $Y$, other equilibria are given by

$x_{1}=\cos \alpha$,

$x_{2}=\sin \alpha$,

$x_{3}=\cos \alpha-\frac{\omega}{\sigma} \sin \alpha$.

$x_{4}=\frac{\omega}{\sigma} \cos \alpha+\sin \alpha$.

$x_{s}=1$.

The transformation $X \rightarrow X \exp (i \alpha)$. $Y \rightarrow Y \exp (i \alpha), \quad Z \rightarrow Z \quad$ corresponds to $x_{1} \rightarrow x_{1} \cos \alpha-x_{2} \sin \alpha_{.} x_{2} \rightarrow x_{1} \sin \alpha+x_{2} \cos \alpha$. $x_{3} \rightarrow x_{3} \cos \alpha-x_{4} \sin \alpha, x_{4} \rightarrow x_{3} \sin \alpha+x_{2} \cos \alpha$. $x_{5} \rightarrow x_{9}:$ thus the rotation matrix in this case can be written down as 
$R(\alpha)=\left(\begin{array}{ccccc}\cos \alpha & -\sin \alpha & 0 & 0 & 0 \\ \sin \alpha & \cos \alpha & 0 & 0 & 0 \\ 0 & 0 & \cos \alpha & -\sin \alpha & 0 \\ 0 & 0 & \sin \alpha & \cos \alpha & 0 \\ 0 & 0 & 0 & 0 & 1\end{array}\right)$

and the methods previously described can be used to study the bifurcation of the limit cycle solution of (3.44): the algebra involved is somewhat tedious, and is not pursued here. However, we can already draw some conclusions from (3.47), (3.32) and (3.37).

In the rotating frame. the components of $x$ will bifurcate to a small amplitude oscillation about a large scale oscillation of much smaller frequency. This follows from (3.32), and is essentially observed in fig. 2 . In the fixed frame, we wili see the same picture, but with the discrepancy in frequencies less visible. However, note that since. from (3.47), we must have $R_{5 ;} x_{0 j}=x_{0 \%}$ and similarly for $U_{n}$, the $z$-component of $z$ is given in either fixed or rotating frame by

$Z=x_{s}=x_{0 s}+\epsilon\left\{A(\tau) \mathrm{e}^{i n t}+(*)\right\}+O\left(\varepsilon^{2}\right) ;$

therefore, the solution for $z$ is singly periodic at bifurcation. and no slow variation of large amplitude should exist: this is seen in fig. 6 .

\section{Numerical results and conclusions}

In the previous sections, we have shown that. except for the singular case $e+r_{2}=0$, only the origin is a fixed point. The two further fixed points of the real Lorenz equations which exist when $r>1$ are replaced by the limit cycle which has frequency $w$. Much of this paper has been devoted to the stability of this limit cycle and the nature of the bifurcation when it becomes unstable. The analysis of section 3 showed that the sign of $\operatorname{Re}\left(k_{2}\right)$ effectively determines whether this bifurcation is of a subcritical
$\left(\operatorname{Re}\left(k_{2}\right)>0\right)$ or supercritical nature $\left(\operatorname{Re}\left(k_{2}\right)<0\right)$ with a transition io doubly periodic motion in the latter case. As McLaughlin and Martin [6] have shown for the real Lorenz equations, the case $\operatorname{Re}\left(k_{2}\right)>0$ is a sub-critical Hopf bifurcation. In our problem the actual determination of the criterion $\operatorname{Re}\left(k_{2}\right)=0$, giving the exact delination between the two types of behaviour, is extremely messy to calculate. Instead we have performed a limited number of numerical integrations to examine the kind of bifurcations which can occur.

There are five parameters in the equations (1.1): $\sigma, b, r_{1}, r_{2}$, and $e$. We will concern ourselves mainly with holding these fixed, except for $r_{1}$, which is thus the bifurcation parameter. Clearly a complete numerical analysis of $(1.1)$ is out of the question in a paper of the present nature, and we can only give a brief idea of the types of bifurcation which occur.

In fig. 3, we show a stability diagram in $\left(r_{1}, r_{2}\right)$ space which shows the dependence of $r_{\mathrm{ls}}$ and $r_{\mathrm{c}}^{\prime}$ on $r_{2}$ at $\sigma=2, e=3 r_{2}, b=0.8$. These represent the analytic results of sections 2 and 3 . Thus to the left of $A$, the origin is linearly stable; to the right, it is unstable and the stable solution is a limit cycle whose frequency decreases to zero

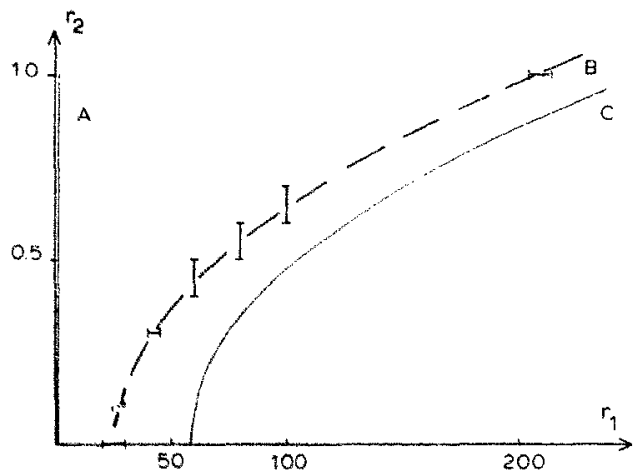

7is. 3. Stabihty diagram in $\left(r_{i}, r_{2}\right)$ space sioving $r_{i s}, r_{k}^{t}$, and also the approximate region of sur, itical transition. Parsmeter values are $b=0.8, \sigma=2, e=3 r$. 
as $r_{2} \rightarrow 0$. This limit cycle is linearly stable beiween $A$ and $C$. and linearly unstable below $C$. Fig. 4 shows the decisy of a trajectary in the rotating ( $\operatorname{Re} x, \operatorname{Re} y$ ) phase space at a value of $r_{1}=354$ (here $r_{i c}^{\prime}=25567 \ldots$ at $\left.r_{2}=1\right)$ : motion is trom top to bottom. an: consints of a fast oscillation of slowly decaying zmplitude, which slowly precesses around the umbrlying limit cycle (set of eyui ibria).

At a value of $r_{1}=256>\cdots$ a small perturbation to the linit cycle initially grows as predicted by the anaysis. but the final resultant motion is hardly of small amplitude, though in other respects it resenbles the result of a supereritical bifurcation. Later stages in the evolution of a small perturbation to the linit cycle ate shown in fig. 5. The motion is anticlockwise. In fig. Sa, we see that from $t=835$ to $t=935$. the solution precesses slowly around the underlying limit cycle, with a precession rate that slowly increases. Fig. $5 \mathrm{~b}$ shows the solution from $t=900$ to 1,000 : the bottom of $5 \mathrm{a}$ is visible on the inside (though the scale is different): we see that the precession rate increases dramatically (more than three complete precessions are visible) and the solution rapidly attains a steady form, which is shown in fig. Sc. Correponding plots of $\operatorname{Re}(x), \operatorname{Re}(y)$ and $z$ are shown in fig. 6 .

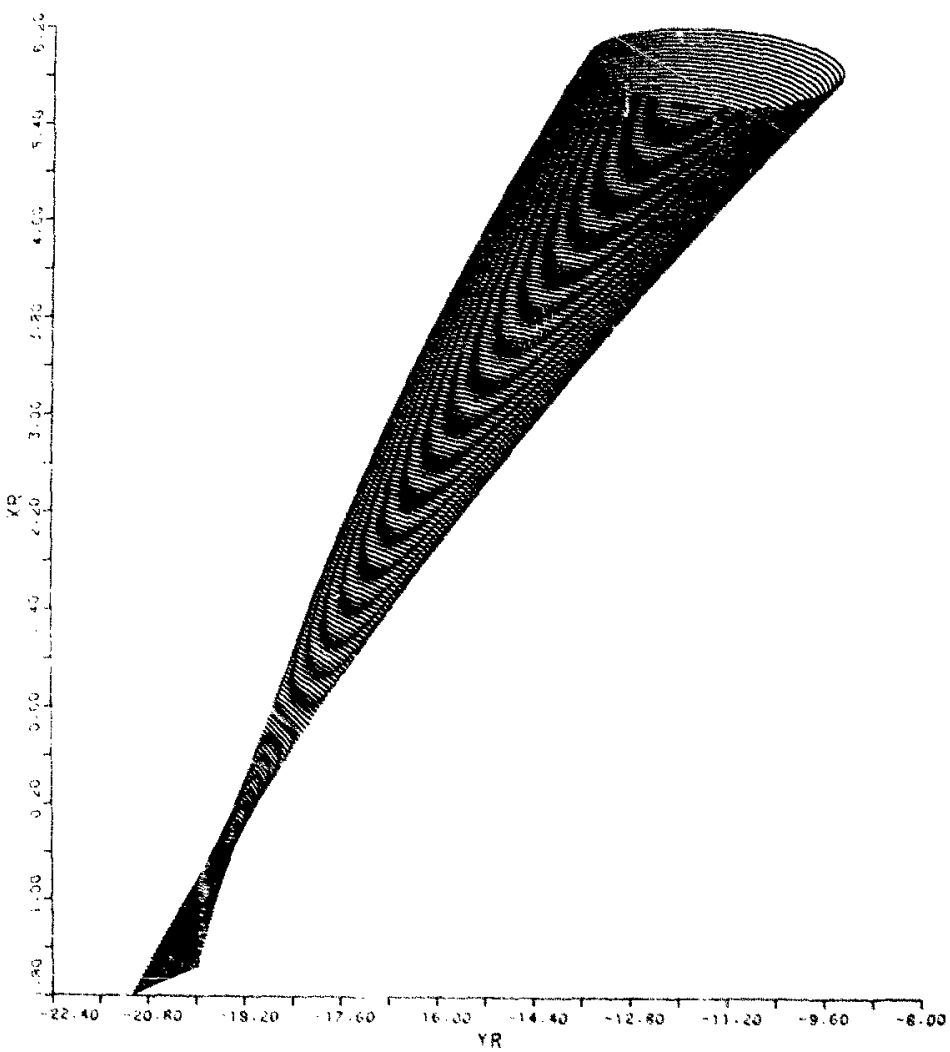

Fig. 4. Slowly decaying trajectory on $\left(r_{R}, y_{R}\right)$ phase space at $n_{1}=254<r_{k}=255.67, \ldots, r_{2}=1 ;$ other patameters as for fig. 3 . 
Fig. 6 shows that between $t=50$ and $t=100$ (different initial values were chosen so that the final steady solution was upproached rapidly, in $t<50$ ) ten full precessions occur, and there are about eight to nine fast oscillations per precession. Close examination of the time plots suggests that (as fig. $5 \mathrm{c}$ also indicates) the solutions are periodic (or very nearly so) with a period of forty-two fast oscillations, or five precession:s.

The large amplitude of this oscillation suggests that the bifurcation at $r_{1}=r_{1 c}^{\prime}=$ $255.67 \ldots$ is sub-critical, and so $\operatorname{Re} k_{2}>0$ there. In this case, we suggest that at values $r_{1}<r_{k}$, there may exist an unstable and a (larger) stable torus, for which the amplitude of the smaller tends to zero as $r_{1} \rightarrow r_{1}^{t}$. Generally, the nature of the stable large-amplitude solution is inaccessible to analysis, but we suggest on the basis of fig. $5 c$ (and other rumerical evidence) that it also consists of motion on a torus which one might expect to be doubly periodis. As $r_{1}$ decreases to a value $r_{\mathrm{k}}^{\prime \prime}<r_{\mathrm{k}}$, the stable and unstable forms may coalesce and vanish: or, they may both be unstable. The dashed line marked $B$ in fig. 3 represents (roughly) the

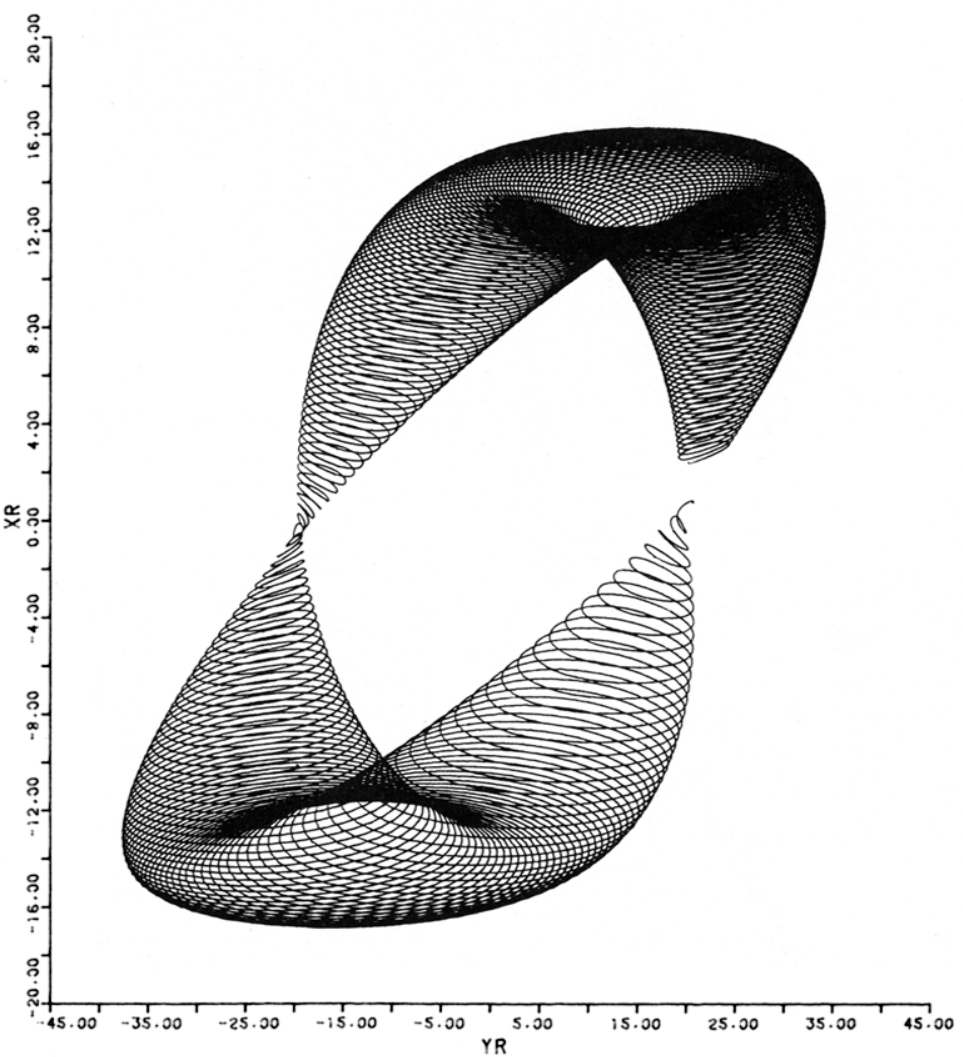

Hig. S. Evalution of small disturbance to the fimit cycle at $r_{1}=256>r_{w}^{\prime}$; other parameters as for fig. $4 ;$ (a) $t=835$ to 935 . 


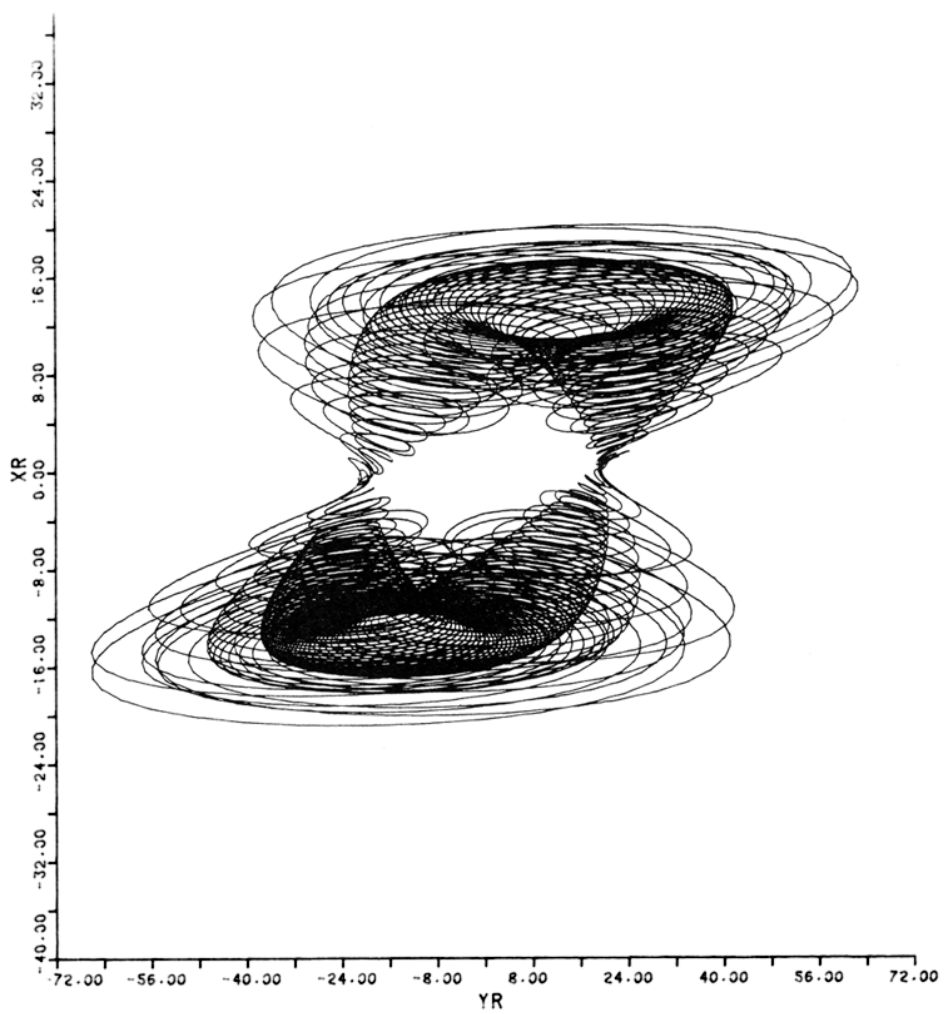

Fig. 5 . (h) $t=900$ to 1.000 .

position of the graph of $r_{\%}^{\prime \prime}$ as a function of $r_{3}$. It has been determined on the hasis of a limited number of integrations. As long as $B$ lies above C. the bifurcation will then be suhcritical. If $B$ intersects $C$, then at this point $\operatorname{Re}\left(k_{2}\right)=0$, and for greater values we should expect $\operatorname{Re}\left(k_{2}\right)<0$, and a supercritical bifurcation: we have not determined this coefficient. however. Although the motion exhibited in fig. 6 is not of small anplitude, the nature of the numerical solution nevertheless suggests a multiple time scales analysis. One possibility for such an analysis is that the parameters are 'close' to the values at wtich $k_{2 R}=0$ : an analysis near this point (and fo: $\left.\left|r_{1}-r_{i}\right|<1\right)$ would describe the oititer stable oscillation as well: on the other hand its form may be due to the largeness of $r_{1}$.

Fig. 7 exhibits the finite amplitude stable torus at a subcritical value of $r_{1}=220<r_{\text {; }}^{*}$. As far as can be seen, the motion is doubly periodic (frequency locking does not occur): the occurrence of precisely two incommensurate frequencies may be (and has been) checked by spectral analyses, and by computing phase plots $o:\left.X\right|^{2}$ versus $|Y|^{2}$ (which appear as limit cycles in a doubly periodic motion).

We have found further bifurcations of familiar type, by decreasing $r_{2}$. Of the twc possibilities, we have found no further instabil ty of the 2-torus to a higher dimensional attracto b't 


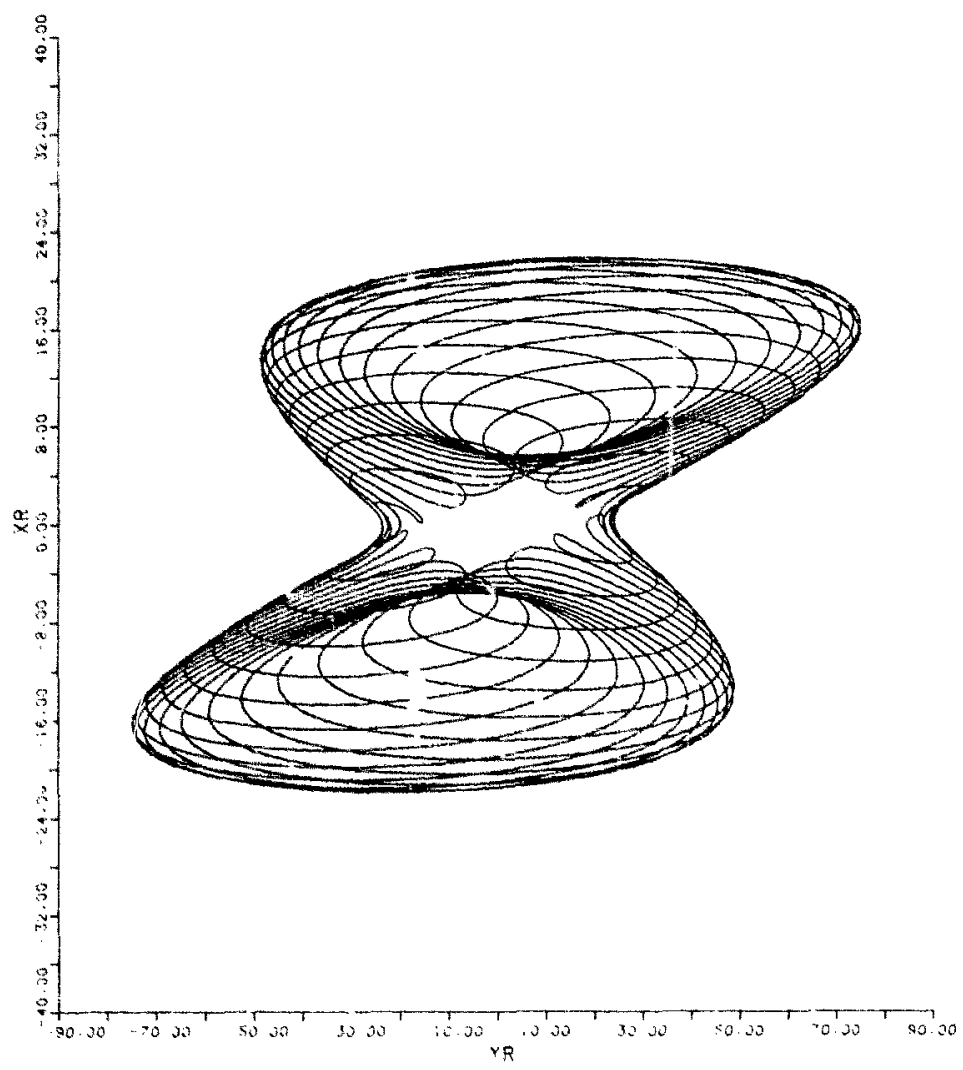

Fig. 5. (c) Final motion, $1 \rightarrow \infty$.

we have found an approach to 'chaos' by 'period-doubling' of the torus; specifically, $|X|^{2}$ versus $|Y|^{2}$ (or $Z$ ) phase plots exhibit perioddoubling limit cycles as $r_{2}$ is reduced at, for example, $r_{1}=40$. This is analogous to the period-doubling approach to chaos in the real Lorenz equations as $r_{1}$ is reduced from infinity (Robbins, 1979). Fig. 8 shows an example of a slightly supercritical $\left(r_{1}>r_{i c}^{\prime}\right)$ chaotic motion (in the rotating frame) at values $r_{1}=60, r_{2}=0.02$, close to the real Lorenz transition to chaos (at $r_{1}=58, r_{2}=0$ ).

Generally speaking, the effect of the cori- plexification is to convert oscillatory states to 'doubly' oscillatory ones: limit cycles into tori, fixed points into limit cycles. Thus increasing the dimension of the system effectiveiy increases the dimension of the attractors, and there is no particular reason to suppose the rich behaviour of the real Lorenz equations is otherwise modified, except in this manner: fuller numerical investigation is necessary for the corroboration of this statement. The chaotic behaviour in the real Lorenz model does not, however, appear to extend very far into the parameter space of the complex Lorenz equa- 

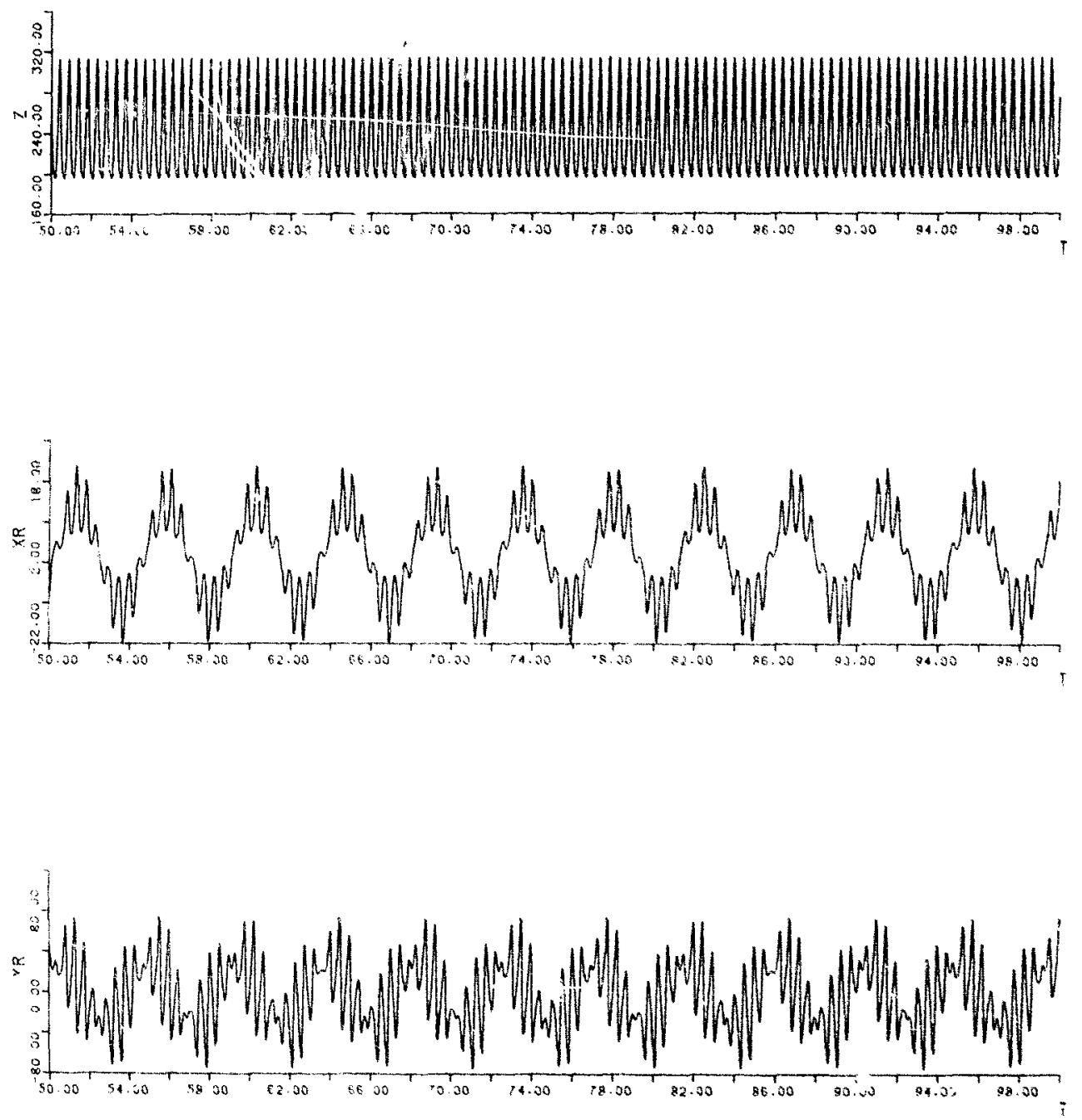

Fig. 6. $x_{R}, y_{k}$ and $z$ plots versus time for fig. $5 c$. 


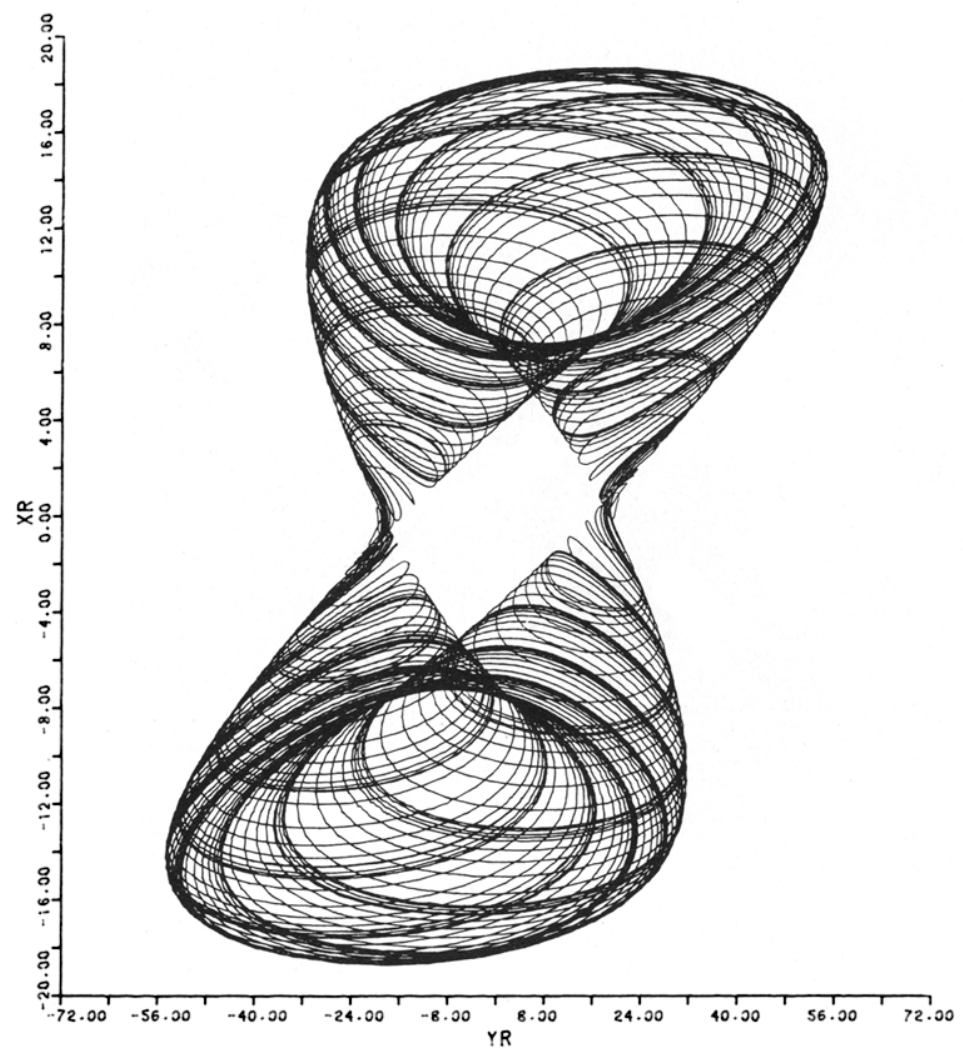

Figure 7. Nonperiodic solution at $\gamma_{1}<r_{\mathrm{k}}$ : same parameters as fg. 2 . 


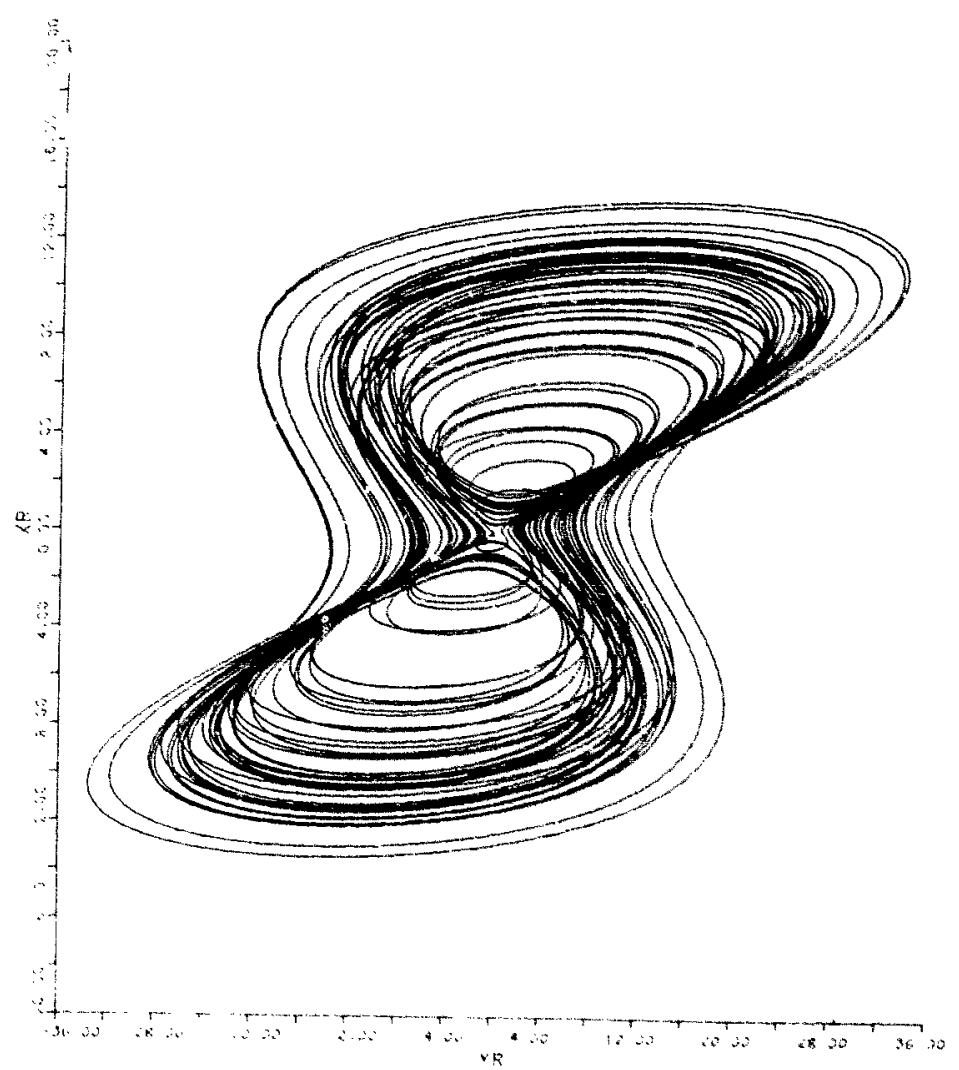

Fig. 8. Chatic motion at $r_{1}=60, r:=0.02$ : other parameters as fig 3 . 
tions, at least when $e=3 r_{3}$. The general tendency of complexifying the equations at fixed $r_{1}$ is thus to replace chaotic behaviour by motion on a torus or a limit cycle.

\section{Acknowledgements}

We are grateful to Joel Smoller and Bob Williams for some very interesting conversations. One of us (MJM) was supported by the Department of Education in Dublin on a Postdoctoral Fellowship. We would also like to express our gratitude to colleagues at Dublin Institute for Advanced Studies School of Theoretical Physics where we held some 'teach your'elf' seminars and where this work was originally started.

\section{References}

[I] J.D. Gibbon and M.J. McGuinness; preprint (1980): to appear in Physica $D$.

[2] E.N. Lorenz. A. Atmos. Sci. 20 (1963) 130.

[3] J.D. Gibbon. M.J. McGuinness and I.N. James. Amplitude equations at the critical roints of unstable dispersive systems, to appear in Proc. Roy. Soc. Series A. (1981).
[4] J.D. Gibbon and M.J. MoGuinness, Thys. Leits. 77A (1980) 295.

[5] J. Brindley and I.M. Moroz, preprint 1980; also Phys. Letts. 77 A (1980) 441 .

[6] J.B. McLaughlin and P.C. Martin, Phys. Rev, A. 12 (1975) 186.

[7] E.A. Coddington and N. Levinson, Theory of Ordinary Differential Equations (McGraw-Hill, New York, 1955).

[8] D. Ruelle and F. Takens, Comm. Math, Phys. 20 (1971) 167.

[9] D.D. Joseph. The bifurcation of T-periodic solutions into $n T$-periodic solutions and TORI, in Synetgetics-A Workshop, H. Haken, ed. (Springer, New York, 1977).

[10] O. Lanford III, Bifurcation of periodic solutions into invariant tori: the work of Ruelle and Takens, in Nonlinear Problems in the Physical Sciences and Biology, Lecture Notes in Mathematics (Springer Verlag, New York) 322 (1977) 159.

[11] J.T. Stuart. J. Fluid Mech, 9 (1960) 353.

[12] H. Haken, Z. Physik B29 (1978) 61.

[13] W. Eckhaus. Studies in Nonlinear Stability Theory, (Springer, New York, 1965).

[14] A.H. Nayfeh, Perturbation Methods (Wiley, New York, 1973).

[15] H. Fujisaka and T. Yamada, Phys. Lett. 66A (1978) 450.

[16] C. Escher, Ber. Buns. Gesell. Phys. Chem 84 (1980) 387.

[17] P.R. Fenstermacher, H.L. Swinney and J.P. Giollub. J. Fluid Mech. 94 (1979) 103.

[18] K.A. Robbins, Siam J. Appl. Math. 36 (1979) 457.

[19] S.S. Hassan. P.D. Drummond and D.F. Walls, Optics Comm. 27, (1979) 480.

[20] K. Ikeda, Optics Comm. 30 (1979) 257.

[21] R. Bonifacio and L.A. Lugiato, Phys. Rev. AlB (1978) 1129. 